THALITA SOUSA DE PAULA

\title{
RESPOSTA PÓS-EXERCÍCIO VISTA NA RESSONÂNCIA NUCLEAR MAGNÉTICA DO MÚSCULO QUADRICEPS EM MULHERES PÓS-MENOPÁUSICAS COM E SEM OSTEOPOROSE
}

Dissertação apresentada à Faculdade de Medicina da Universidade de São Paulo para obtenção do título de Mestre em Ciências.

Programa de Fisiopatologia Experimental

Orientadora: Profa. Dra. Júlia Maria D’Andrea Greve

(Versão corrigida. Resolução CoPGr 6018, de 03 de outubro de 2011. A versão original está disponível na Biblioteca FMUSP)

SÃO PAULO 
THALITA SOUSA DE PAULA

\section{RESPOSTA PÓS-EXERCÍCIO VISTA NA RESSONÂNCIA NUCLEAR MAGNÉTICA DO MÚSCULO QUADRICEPS EM MULHERES PÓS-MENOPÁUSICAS COM E SEM OSTEOPOROSE}

Dissertação apresentada à Faculdade de Medicina da Universidade de São Paulo para obtenção do título de Mestre em Ciências.

Programa de Fisiopatologia Experimental

Orientadora: Profa. Dra. Júlia Maria D’Andrea Greve

(Versão corrigida. Resolução CoPGr 6018, de 03 de outubro de 2011. A versão original está disponível na Biblioteca FMUSP)

SÃO PAULO 
de Paula TS. Resposta pós-exercício na ressonância nuclear magnética do músculo quadríceps em Mulheres pós-menopausa com e sem osteoporose [mestrado]. São Paulo: Universidade de São Paulo, Faculdade de Medicina; 2018.

Aprovado em:

Banca Examinadora

Prof. Dr.:

Instituição:

Julgamento:

Prof. Dr.:

Instituição:

Julgamento:

Prof. Dr.:

Instituição:

Julgamento: 


\section{DEDICATÓRIA}

Aos meus pais, Moacir Aparecido de Paula (in memorian) e Maria Áurea Batista de Sousa por me mostrarem a importância da família e por sempre terem me incentivado aos estudos.

Aos meu irmãos Alessandra Sousa de Paula Benitez e Marco Aurélio Sousa de Paulo e cunhados Douglas Vaz Benitez e Elaine Costa de Paulo pelo companheirismo e todo apoio sempre!

Aos meus amados sobrinhos Gustavo de Paula Benitez, Rafael de Paula Benitez e Matheus Costa de Paulo, por fazerem do meu mundo mais alegre e pela compreensão, muitas vezes mesmo sem saber, dos momentos que deixei de estar com eles para me dedicar a esta dissertação. 


\section{AGRADECIMENTOS}

À Deus por me guiar e iluminar durante toda minha vida e por me sustentar com força e coragem a cada novo obstáculo que surgia nesta longa caminhada.

À minha família pela compreensão, companheirismo e incentivo sempre depositados em mim.

Meu agradecimento especial à minha orientadora Prof ${ }^{\mathrm{a}}$ Dra. Júlia Maria D'Andrea Greve, Coordenadora do Laboratório de Estudos do Movimento do IOT-HC/FMUSP, não só pela brilhante orientação, mas também por ser um exemplo como pessoa e profissional.

Ao Prof. Guilherme Carlos Brech pela co-orientação, paciência e por toda sua dedicação e contribuição para que essa dissertação acontecesse.

Aos professores do Departamento de Ginecologia e Obstetricia-HC/FMUSP, Prof. Dr. Edmund Chada Baracat, Prof. Dr. José Maria Soares Júnior pelo apoio no início do projeto e a Profa. Dra. Sylvia Hayashida pela ajuda nas triagens realizadas em seus ambulatórios.

Aos biomédicos Thiago Antônio Fedele e Meriellem Galvão Masseli pela essencial ajuda na realização e análise dos exames de Ressonância Magnética.

À todos os funcionários do Laboratório de Estudo do Movimento LEM-IOT/HCFMUSP, em especial ao André Silva por toda disponibilidade e também ao Marcelo Pedro pela ajuda nos testes do dinamômetro isocinético.

À secretaria do programa de Fisiopatologia Experimental, em especial ao funcionário Igor Tolgyesi pela atenção dispensada, principalmente na reta final desta pesquisa.

À minha querida amiga-irmã Débora Pedrolo Parisi pelo incentivo para que eu iniciasse nn programa de pós-graduação.

Às minhas best amigas Aline Novello, Tamiris Silva, Daysi Tobelem e Fernanda Cordeiro pelas contribuições que cada uma de algum modo prestou e pela torcida durante todas as etapas. 
Às pacientes e voluntárias desta pesquisa, sem as quais não seria possível realizá-la.

Agradeço a todos que contribuíram direta ou indiretamente na condução deste projeto e que cometi a indelicadeza de não mencionar nominalmente.

A todos que acreditam e acreditaram em mim... 


\section{RESUMO}

A menopausa é o final da vida reprodutiva da mulher e pode ter como consequência a perda da massa óssea e desenvolvimento da osteoporose. A sarcopenia decorrente do processo de envelhecimento acarreta na diminuição de massa e força muscular, déficit de desempenho e maior risco de quedas e fraturas. A Ressonância Nuclear Magnética (RNM) é uma ferramenta não invasiva e eficaz para a avaliação quantitativa e da dinâmica metabólica do músculo esquelético. Por meio do mapa T2 é possível captar as alterações musculares agudas causadas pela atividade física. A intensificação do sinal T2 é causada pelo movimento osmótico da água intramuscular, aumento da acidose e do volume do espaço intracelular. O objetivo desta pesquisa foi avaliar a influência da densidade mineral óssea no metabolismo muscular de mulheres pós-menopáusicas. Foram avaliadas 16 pacientes do sexo feminino, no período pósmenopausa há mais de 12 meses, com média de idade de 63 anos, divididas em GrupoOsteoporose $(\mathrm{GO}=9)$ e Grupo Controle $(\mathrm{CG}=7)$. Todas foram submetidas ao exame de Ressonância Nuclear Magnética da região da coxa (RNM1) e em seguida fizeram uma dinamometria isocinética na velocidade de 180 graus/segundo (duas séries de 10 contrações voluntárias máximas) e exercícios específicos para ativação do músculo quadríceps (agachamento e "step"), e após os exercícios, fizeram a RNM2. Os resultados mostraram aumento do mapa T2, caracterizado pelo maior tempo de relaxamento nos dois grupos avaliados, sem diferença entre eles. Não se observou correlação significativa dos resultados da RNM2 com os parâmetros de força (pico de torque corrigido pela massa corporal) e potência (trabalho total das 10 repetições da segunda série) e com a dosagem de vitamina D. Também não houve correlação entre a dinamometria isocinética e dosagem de vitamina D. A osteoporose não afeta a resposta muscular do quadríceps ao exercício, avaliada pelo mapa T2 da ressonância nuclear magnética. A metodologia é robusta e eficiente, mostrando que a RM é um método sensível para medir mudanças metabólicas no músculo após o exercício.

Palavras-chave: Osteoporose, Pós-Menopausa, Sarcopenia, Fadiga muscular, Imagem por Ressonância Magnética 


\section{ABSTRACT}

Menopause is the end of woman's reproductive life and consequences as loss of bone mass and osteoporosis may emerge. The ageing's sarcopenia entails the reduction of muscle mass and strength, deficit of physical performance and increases the risk of falls and fractures, which is also present in postmenopausal women. Magnetic resonance imaging (MRI) is a noninvasive and effective tool for quantitative assessment and metabolic dynamics of skeletal muscle. Through the T2 map is possible to capture acute muscle disorders caused by physical activity. Intensification of T2 sign is caused by osmotic movement of intramuscular water, increase of acidosis and intracellular space volume. The aim of this study was to evaluate bone mineral density in muscle metabolism in postmenopausal women. We evaluated 8 female patients in postmenopausal for more than 12 months, with a mean age of 63 years, divided into osteoporosis-group $(\mathrm{GO}=9)$ and control group $(\mathrm{CG}=7)$. They were submitted to MRI examination of thigh at rest (RM1), and then the isokinetic dynamometer at the speed of 180 degrees/second, 2 sets of 10 maximal voluntary contractions and specific exercises to activate the quadriceps muscle (squats and step) and then the RM2 to capture the muscle metabolic changes. For perception of fatigue level, samples of lactate were taken at rest (Lac1), after 1 minute (lac2) and 3 minutes (Lac3) from the end of the exercises. In both groups, it was observed variation of lac2 Lac3, confirming that fatigue levels and changes in RM2 compared to RM1 in the uptake of water were achieved due to intramuscular specific physical changes in post-exercise muscle metabolism. The results showed increased T2 map, characterized by the highest relaxation time in both groups and there are no difference between them. There was no significant correlation of the results of the RNM2 with the parameters of force (peak torque corrected by body mass) and potency (total work) and with the dosage of vitamin D. There was also no correlation between the isokinetic dinamometria and dosage of vitamin D. Osteoporosis does not affect the muscle response of the quadriceps to exercise, assessed by the T2 map of magnetic resonance imaging. The methodology proved to be robust and efficient, showing that MRI is a sensitive method to measure metabolic changes in muscle after exercise.

Keywords: Osteoporosis, Postmenopausal, Sarcopenia, Muscle Fatigue, Magnetic Resonance Imaging 


\section{LISTA DE FIGURAS}

Figura 1. Fluxograma da avaliação e coleta de dados ............................................... 25

Figura 2. Dinamômetro isocinético .................................................................... 27

Figura 3. Exercício de degrau e exercício de agachamento .......................................... 28

Figura 4. Fixação dos marcadores para referência da Ressonância Magnética .................. 29

Figura 5. Exame de Ressonância Magnética ................................................................ 30

Figura 6. RNM 1 - Imagem Ressonância Magnética em repouso. RNM 2 - Imagem Ressonância Magnética pós-exercício 


\section{LISTA DE TABELAS}

Tabela 1 - Descrição física e clínica da amostra dividida em Grupo Osteoporose (GO) e Grupo Controle(GC) (média e desvio padrão)

Tabela 2 - Mapa T2 (Sinal do tempo de relaxamento T2) (média e desvio padrão) da Ressonância Nuclear Magnética (RNM) pré e pós exercícios do Grupo Osteoporose (GO) e Grupo Controle (GC) na coxa direita (D) e esquerda (E)

Tabela 3 - Mapa T2 (Sinal do tempo de relaxamento T2) (média e desvio padrão) da Ressonância Nuclear Magnética (RNM) pré e pós-exercício do Grupo Osteoporose (GO) e Grupo Controle (GC) em ambas as coxas

Tabela 4 - Dinamometria isocinética (média e desvio padrão) de mulheres pósmenopausa com e sem osteoporose

Tabela 5 - Dosagem de $25 \mathrm{OH}$ Vitamina D (ng/ml) (média e desvio padrão) em mulheres pós-menopausa com e sem osteoporose

Tabela 6 - Dosagem de $25 \mathrm{OH}$ Vitamina D (ng/ml) (média e desvio padrão) em mulheres pós-menopáusicas com e sem osteoporose

Tabela 7 - Distribuição dos valores de coeficiente de correlação e valor de p referentes ao trabalho do músculo quadríceps entre os valores do Dinamômetro Isocinético, Ressonância Nuclear Magnética e Vitamina D 


\section{LISTA DE ABREVEATURAS}

Op - Osteoporose

DMO - Densidade Mineral Óssea

RNM - Ressonância Nuclear Magnética

TC - Tomografia Computadorizada

DXA - Densitometria de composição corporal

BIA - Bioimpedância

T2 - Tempo 2 - Tempo de relaxamento transverso

STIR - Resuperação da inversão com tempo curto

CK - Creatinofosfoquinase

IPAQ - Questionário Internacional de Atividade Física

Lac - Lactato

IMC - Índice de massa corpórea

ROI - Região de interesse 


\section{LISTA DE SIGLAS}

$\mathrm{mmHg}$ - milímetros de mercúrio

$\mathrm{kg}$ - quilograma

$\mathrm{cm}$ - centrímetro

$\mathrm{kg} / \mathrm{cm}^{2}$ - quilograma por centímetro quadrado

$\%$ - porcentagem

o - grau

mg - miligrama

dl - decilitro

ng - nanograma

$\mathrm{ml}$ - mililitro

$\mathrm{ng} / \mathrm{ml}$ - nanograma/mililitro

w - watts 


\section{SUMÁRIO}

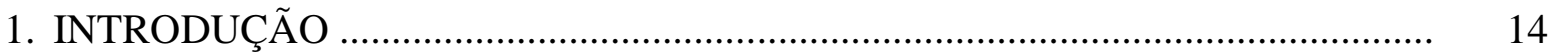

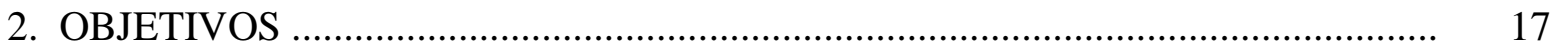

3. REVISÃO DA LITERATURA ……………………................................... 18

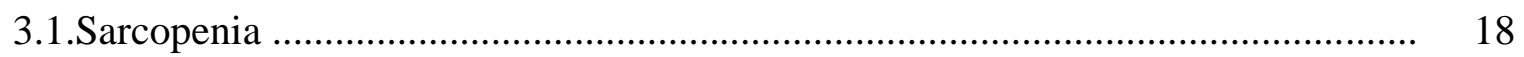

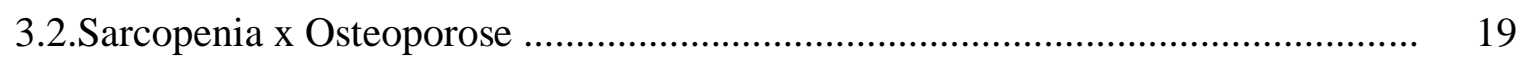

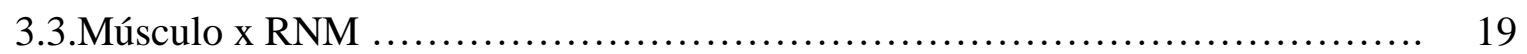

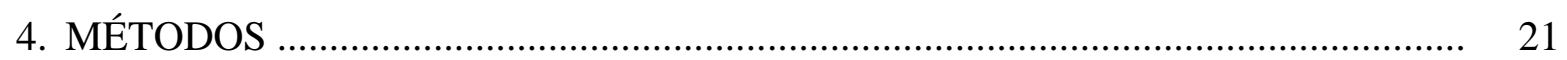

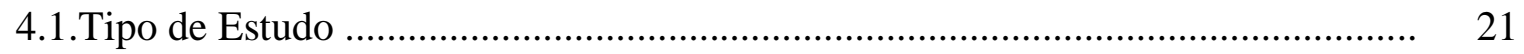

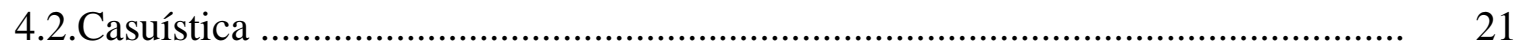

4.3. Critérios de inclusão .............................................................................. 21

4.4. Critérios de exclusão ...................................................................................... 22

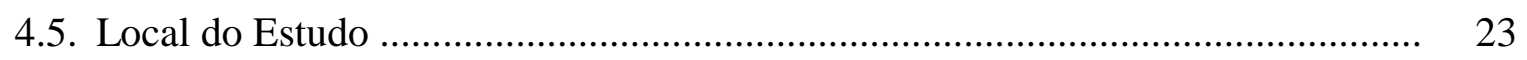

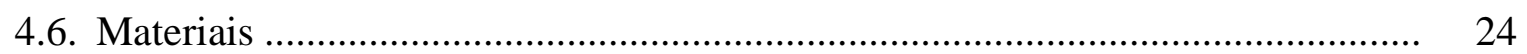

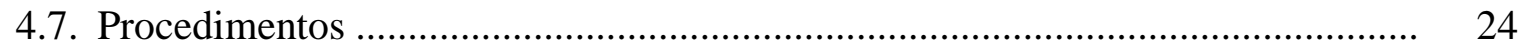

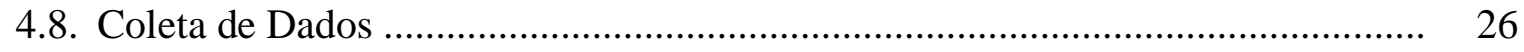

4.8.1.Questionário ................................................................................ 26

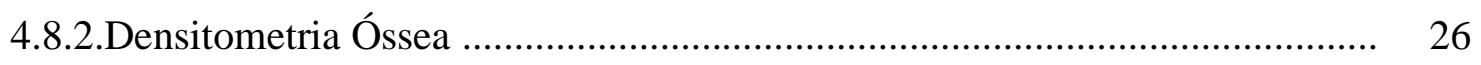

4.8.3. Vitamina D e Cálcio .................................................................................. 26

4.8.4. Protocolo de Fadiga Músculo Quadríceps Femoral ...................................... 27

4.8.4.1.Dinamometria Isocinética dos Flexores/ Extensores Joelho .................. 27

4.8.4.2. Exercícios complementares ................................................................. 27

4.8.5.Dosagem Lactato .............................................................................. 28

4.8.6.Ressonância Nuclear Magnética (RNM) …………………………………... 28

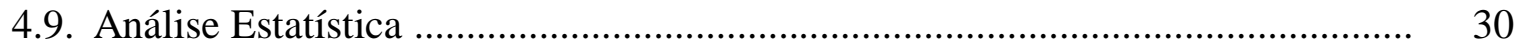

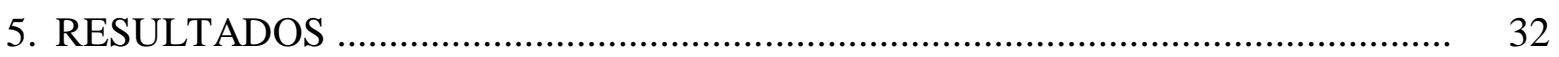

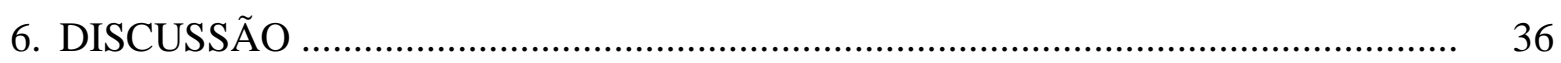

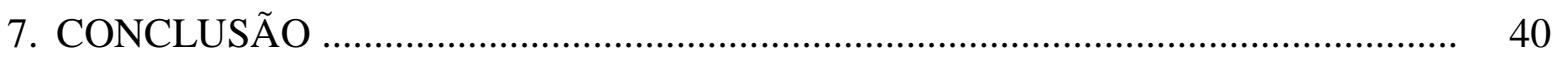

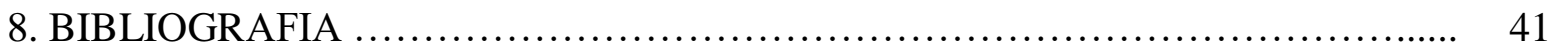

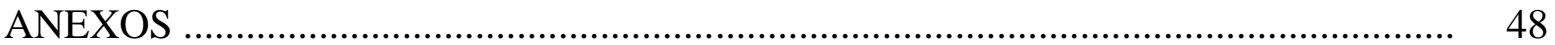




\section{INTRODUÇÃO}

A menopausa é o final da vida reprodutiva da mulher (Velde;Dorland;Broekmans, 1998; Aidelsburger et al., 2012; Roberts;Hickey, 2016) e ocorre, em média, aos 51 anos em países industrializados e aos 48 em países não industrializados (Sapre;Thakur, 2014). O período pósmenopáusico é definido como o período após 12 ou mais meses de amenorreia e é nessa fase que, por alterações metabólicas, surge o estado de hipoestrogenismo que pode ter como uma de suas consequências a diminuição da massa óssea (Doubova et al., 2011; Aidelsburger et al., 2012; Pimenta et al., 2012).

Neste período, segundo o Ministério da Saúde, por meio do Sistema de vigilância de fatores de risco para doenças crônicas, o Vigitel de 2010, 37,3\% das idosas brasileiras, com 65 ou mais anos, são sedentárias (Brasil, 2011), aumentando a predisposição à osteoporose (OP) que trata-se da diminuição da densidade mineral óssea com alteração de sua microarquitetura e aumento do risco de fraturas e/ou deformidades ósseas (NAMS, 2001; Hellekson, 2002; Souza, 2010; Sandhu;Hampson, 2011; Aidelsburger et al., 2012; Bączyk et al., 2012; Carvalho;Kurlak;Borba, 2012; Sapre;Thakur, 2014).

Para o diagnóstico da OP, a Organização Mundial de Saúde (WHO, 1994) recomenda a realização do exame de densitometria óssea, sendo o padrão de referência da densidade mineral óssea (DMO) em mulheres pós-menopáusicas ou acima de 50 anos dado pelo T-Score (comparação da DMO do indivíduo que está realizando em relação com a massa óssea de mulheres jovens durante o período reprodutivo). Assim, a osteoporose será expressa pelo TScore menor ou igual a $-2,5$ desvios-padrão em relação à média das mulheres jovens, a osteopenia pelo T-Score entre -1,0 e -2,5 desvios-padrão e a DMO será considerada normal se T-Score maior ou igual a -1,0 desvios-padrão (Souza, 2010; Sandhu;Hampson, 2011; Maghraoui et al., 2012; Arantes et al., 2016).

Outra alteração bastante comum neste período é a deficiência de vitamina D, expressa pelos níveis plasmáticos de 25-hidroxi vitamina D $[25(\mathrm{OH}) \mathrm{D}]$, que por suas funções na interação entre músculos e ossos causa diminuição da DMO e sarcopenia, termo que pode ser definido como perda de massa muscular com consequentes deficiências de desempenho físico (Pedrosa;Castro, 2005; Cranney et al., 2008; Bandeira et al., 2010; Carvalho;Kurlak;Borba, 2012; Bączyk et al., 2012; Maghraoui et al., 2012; Go et al, 2013; Azzabou;Hogrel;Carlier, 2015; Gunton et al., 2015; Phu;Derek;Duque, 2015; Arantes et al., 2016; Kühn;Trotz;Stangl, 2018). 
Para detecção de tais alterações advindas da sarcopenia, o dinamômetro isocinético mostra-se como instrumento padrão ouro de avaliação de força muscular, sendo o método mais utilizado na avaliação da função e do desempenho muscular (Aquino;Leme, 2006; Zabka;Valente;Pacheco, 2011).

Outro instrumento, mais recente, que tem sido eficaz para a avaliação muscular é a ressonância nuclear magnética (RNM), pelas medidas dos valores quantitativos da dinâmica metabólica do músculo esquelético durante condições degenerativas, respostas inflamatórias, processos regenerativos e após exercícios (Esposito et al., 2013, Cruz-Jentoft et al, 2010; Martins-Bach et al., 2015).

A RNM fornece imagens do recrutamento dos compartimentos musculares, dadas pelo aumento da intensidade do Sinal de Tempo 2 (T2), mostrando padrões espaciais e de intensidade da ativação do músculo. As alterações no sinal de T2, vistas no chamado mapa T2, são causadas pelo aumento agudo da atividade muscular e sua relação com o tempo de relaxamento de T2, que depende da água presente no músculo. A intensificação do sinal T2 é causada pela motilidade osmótica dos prótons de água intramuscular que entram nas células por causa do aumento da acidose e do volume do espaço intracelular, causados pelos catabólitos musculares (Prior et al., 1999; Meyer;Prior, 2000; Patten;Meyer;Fleckenstein, 2003; Esposito et al., 2013, Martins-Bach et al., 2015). Assim o tempo de movimentação da água intramuscular e sua repercussão no metabolismo muscular podem ser medidos e servirem com parâmetros da ativação muscular.

Além da perda de fibras musculares no processo de sarcopenia, ocorrem infiltrados gordurosos dentro e entre as fibras musculares (Esposito et al., 2013; Phu;Derek;Duque, 2015) e a quantidade de gordura é um fator complicador para a avaliação das imagens da RNM (Ribeiro et al., 2013). Para minimizar o sinal da gordura no músculo, utiliza-se uma sequência STIR que fornece uma informação qualitativa sobre o tônus e edema muscular e infiltrados gordurosos (Ribeiro et al., 2013, Sciorati et al., 2014).

Não foram encontrados, na literatura disponível, pesquisas que avaliaram a ativação muscular do músculo quadríceps em mulheres pós-menopáusicas, porém existem estudos que mostram a RNM como um método diagnóstico eficaz e não invasivo de avaliação muscular. Desta forma, o mapa T2, pode ser indicado para visualizar e quantificar aspectos da função muscular, como as afecções agem nos padrões de ativação muscular e as adaptações dos músculos aos exercícios (Prior et al., 1999; Meyer;Prior, 2000; Patten;Meyer;Fleckenstein, 2003; Tawara et al., 2011; Sousa et al., 2012; Esposito et al., 2013; Martins-Bach et al., 2015). 
As ferramentas não invasivas para avaliação muscular têm aplicação imediata em protocolos terapêuticos com possíveis benefícios nos estudos longitudinais, pelo impacto mínimo sobre os indivíduos. Para isso, é essencial que a ferramenta selecionada possa detectar alterações patológicas e o comportamento dos músculos normais. (Martins-Bach et al., 2015). O padrão de imagem de RNM da ativação do músculo quadríceps de mulheres com osteoporose foi avaliado pela primeira vez, no atual estudo.

A hipótese levantada, no presente estudo, é de que a diminuição da densidade mineral óssea pode influenciar no padrão de ativação muscular e que a identificação desta relação pode ser útil para o estabelecimento de programas mais efetivos de exercícios para prevenção e tratamento da osteoporose e suas consequências. 


\section{OBJETIVOS}

\section{Geral}

Avaliar a influência da densidade mineral óssea na atividade muscular do músculo quadríceps de mulheres pós-menopáusicas.

\section{Específicos}

Avaliar a correlação entre a:

- Densidade mineral óssea e atividade muscular em mulheres pós-menopáusicas vista na ressonância nuclear magnética.

- Dosagem de vitamina D e a atividade muscular em mulheres pós-menopáusicas vista na ressonância nuclear magnética.

- Dosagem da vitamina D e força muscular medida na dinamometria isocinética em mulheres pós-menopáusicas. 


\section{REVISÃO DA LITERATURA}

\subsection{Sarcopenia}

A sarcopenia é a diminuição de massa e força muscular e desempenho físico causada pelo envelhecimento (Azzabou;Hogrel;Carlier, 2015; Phu;Derek;Duque, 2015). Kawao e Kaji (2015), em artigo de revisão, afirmam que as mulheres pós-menopáusicas sofrem alterações na composição corporal por perda de massa muscular e aumento de gordura, mudanças que estão relacionadas com a diminuição dos níveis de estrogênio.

A sarcopenia é uma das alterações decorrentes do envelhecimento e devido aos déficits funcionais que ela acarreta, pode levar o idoso a ser a considerado como frágil, conceito que vem sendo bastante utilizado quando trata-se de estudos com idosos. A Síndrome da Fragilidade é, por tanto, uma situação que gera declínio de energia e é identificada por cinco critérios: 1) perda de peso não intencional; 2) diminuição da força (avaliada pela preensão manual); 3) baixa resistência a fadiga; 4) baixo nível de atividade física e 5) diminuição da velocidade de caminhada. (Fried et al. 2001)

Para mensuração da sarcopenia, o Consenso Europeu de Sarcopenia de 2010 e descreve o uso de ressonância nuclear magnética (RNM), tomografia computadorizada (TC), densitometria de composição corporal (DXA) e análise de bioimpedância (BIA), e ressalta RNM e a TC como padrões ouro para esta avaliação, a DXA como o método mais utilizado tanto em pesquisas como clincamente e a BIA como um método barato e que oferece a estimativa de volume de gordura e massa magra corporal. Apesar de existirem muitos intrumentos para avaliação (Cruz-Jentoft et al, 2010; Cruz-Jentoft;Landi, 2014).

Ainda que a sarcopenia seja comum e tenha enormes custos pessoais e sociais, não há evidências sobre quais parâmetros são melhores para medir a função muscular (CruzJentoft;Landi, 2014). Reijnierse et al. (2015) referem que não há consenso nos critérios de diagnóstico da sarcopenia, estes autores realizaram um estudo transversal usando como critérios de diagnóstico de sarcopenia: massa muscular relativa (densitometria de composição corporal), massa muscular absoluta (análise de bioimpedância), força muscular (“handgrip”) e desempenho físico (teste de caminhada de seis minutos). Foram avaliados dois grupos: 308 idosos saudáveis e fisicamente ativos e 123 idosos institucionalizados com histórico de quedas e deficiência de equilíbrio. Os autores não conseguiram estabelecer um padrão para as taxas de 
sarcopenia dentro da mesma população e referem variações entre os critérios de diagnósticos utilizados.

\subsection{Sarcopenia x Osteoporose}

É possível identificar uma relação significativa entre a sarcopenia e a osteoporose, situação descrita por Di Monaco et al. (2011) que avaliaram 313 mulheres com histórico de fratura de quadril com a densitometria óssea e cálculo da massa magra do esqueleto apendicular.

Segundo Tarantino et al. (2013), a osteoporose e a sarcopenia são as alterações musculoesqueléticas que mais afetam a população idosa, especialmente as mulheres. A diminuição da microarquitetura do tecido ósseo causada pela osteoporose e a diminuição do tamanho e quantidade de fibras musculares, principalmente do tipo II, da sarcopenia aumentam o risco de fraturas e são as maiores responsáveis pelas limitações funcionais e dependência física nesta população. Estes autores, também, afirmam que a interação osso-músculo é o ponto de partida para o desenvolvimento de instrumentos adequados para diagnóstico e desenvolvimento de terapias que melhorem a qualidade do osso, trofismo muscular e qualidade de vida de idosos frágeis.

Quando tais achados são associados com a dosagem de vitamina D, é possível encontrar uma estreita interação entre osso e músculo na patogênese da osteopenia/osteoporose e sarcopenia, com piora da funcionalidade quando se tem a presença das duas alterações em mulheres pós-menopáusicas (Drey et al., 2015; Kawao e Kaji, 2015; Kühn;Trotz;Stangl, 2018). Tal situação é confirmada devido o déficit de vitamina D causa hipotrofia das fibras tipo II (contração rápida e força) e aumenta o risco de quedas (Glerup et al., 2000; Tarantino et al., 2013; Brincat et al., 2015; Phu;Derek;Duque, 2015; Wu et al., 2016).

Girgis et al. (2015) e Gunton et al. (2015) em seus artigos de revisão, sugerem que a vitamina D age no controle de diversos hormônios, que atuam sobre os ossos e músculos, modulando a relação entre eles.

A suplementação da vitamina D reduz os índices de perda óssea nas mulheres idosas e pode reduzir a velocidade da perda de força muscular, prevenir quedas e consequentemente fraturas, as fraturas osteoporóticas apresentam-se como importante questão na saúde de mulheres pós-menopáusicas. (Bączyk et al., 2012; Maghraoui et al., 2012, Brincat et al., 2015; Kühn;Trotz;Stangl, 2018). 


\subsection{Músculo x RNM}

A RNM é um método eficaz para avaliação das variações dos padrões de ativação muscular, diferenças entre compartimentos musculares e alterações em relação ao ganho de hipertrofia de um músculo ou grupo muscular, assim é possível afirmar que ela é sensível para detectar alterações musculares morfológicas, funcionais e fisiológicas agudas e crônicas, ou seja, a curto e a longo prazos (Mendiguchia et al. 2013; Wakahara et al. 2012; Da Cruz, 2014).

As alterações musculares causadas pela idade foram investigadas por Esposito et al. (2013) que avaliaram músculos de ratos e concluíram que RNM é sensível à resposta inflamatória aguda e permite monitorar modificações sutis na arquitetura e no estado inflamatório do músculo esquelético, como aquelas associadas com a sarcopenia inicial do envelhecimento. Outros estudos, realizados em humanos também mostram a eficácia deste instrumento na detecção de alterações do envelhecimento, porém sem encontrar correlação com prática de atividade física. Há ainda na literatura a desrição da RNM em músculos patológicos, mostrando mais uma aplicação da RNM como uma importante técnica não invasiva para avaliação de resultados em protocolos terapêutcos.

Da Cruz (2014) em estudo desenvolvido no Instituto de Orotpedia e Traumatologia do Hospital das Clínicas de São Paulo - IOT-HC/FMUSP, avaliou as em 12 mulheres saudáveis e ativas após uma sessão exercícios pelo método Pilates, foi realizada a dinamometria isocinética dos extensores do joelho antes e depois da sessão de exercícios. Foram avaliados os níveis séricos de creatinofosfoquinase $(\mathrm{CK})$ e a presença de dor muscular de início tardio pela palpação local. Após os exercícios foi observado da intensidade de sinal T2 nos músculos retofemoral, vasto lateral e glúteo máximo na RNM, com piora do desempenho dos músculos extensores na dinamometria isocinética (pico de torque, trabalho total e potência), presença de dor muscular de 24 a 48 horas após os exercícios e aumento dos níveis séricos da CK após 96 horas. 


\section{MÉTODOS}

\subsection{Tipo de Estudo}

Trata-se de um estudo experimental do tipo longitudinal intervencional, aprovado pela Comissão de Ética para Análise de Projetos de Pesquisa (CAPPesq) da Faculdade de Medicina da Universidade de São Paulo (FMUSP), protocolo número 10785/2013.

\subsection{Casuística}

Foram incluídas 16 pacientes do sexo feminino divididas em dois grupos:

Grupo Osteoporose (GO) - nove pacientes com diagnóstico de osteoporose de acordo com a DMO (T-Score $\geq-2,5$ ) advindas do Serviço de Ginecologia - Setor de Climatério do Instituto Central (IC) do Hospital das Clínicas da Faculdade de Medicina de São Paulo (HCFMUSP) e da comunidade.

Grupo Controle (GC) - sete pacientes sem diagnóstico de osteoporose e osteopenia de acordo com a DMO (T-Score $\leq-1,0)$ advindas da comunidade.

\subsection{Critérios de inclusão}

Ambos os Grupos

1. Estar no período pós-menopáusico com 12 ou mais meses de amenorreia;

2. Idade entre 55 e 65 anos (pós-menopausa tardia);

3. Mulheres que não praticassem atividade física regularmente pelos últimos 3 meses;

4. Sem lesão, doença ou trauma nos membros inferiores nos últimos seis meses;

5. Sem limitações de amplitude articular e / ou deformidades em joelho e quadril;

6. Ser capaz de realizar marcha independente, sem meios auxiliares e sem claudicação por pelo menos 100 metros;

7. Ser independente em suas atividades de vida diária;

8. Não usar marca-passo, ter implantes metálicos ou sofrer de claustrofobia, que impeçam a realização da RNM;

9. Não fazer uso de medicamentos como hormônios da tireoide, estrogênios ou substâncias herbais, antidepressivos e diuréticos; 
10. Ausência de doenças do sistema endócrino: hiperparatireoidismo, diabetes, hipertensão, hipotireoidismo e hiperprolactinemia.

\section{Grupo Osteoporose}

1. Diagnóstico de osteoporose de acordo com a DMO (T-Score $\geq-2,5$ ).

\section{Grupo Controle}

1. Sem diagnóstico de osteoporose e osteopenia de acordo com a DMO (T-Score $\leq-1,0)$

\subsection{Critérios de exclusão}

1. Incapacidade de realizar algum dos testes;

2. Pressão arterial sistólica igual ou acima de $160 \mathrm{mmHg}$ e diastólica igual ou acima de $120 \mathrm{mmHg}$ no momento do teste;

3. Referir dor durante a realização do teste isocinético.

Na Tabela 1, podem ser vistos os dados descritivos da amostra incluída: 
Tabela 1 - Descrição física e clínica da amostra dividida em Grupo Osteoporose (GO) e Grupo Controle(GC) (média e desvio padrão)

\begin{tabular}{lccc}
\hline & GO & GC & p \\
\hline N & 9 & 7 & \\
Idade (anos) & $61,33( \pm 3,57)$ & $61,86( \pm 2,61)$ & $\mathrm{p}=0,746$ \\
Tempo de Menopausa (anos) & $13,2( \pm 3,3)$ & $13,00( \pm 4,83)$ & \\
Massa Corpórea (Kg) & $60,26( \pm 11,89)$ & $69,40( \pm 16,13)$ & $\mathrm{p}=0,212$ \\
Estatura (m) & $1,52( \pm 0,04)$ & $1,55( \pm 0,75)$ & $\mathrm{p}=0,337$ \\
IMC (kg/m $\left.{ }^{2}\right)$ & $25,90( \pm 4,15)$ & $28,34( \pm 3,45)$ & $\mathrm{p}=0,232$ \\
Densitometria óssea & & & $*{ }^{*}<0,001$ \\
$\quad$ L1-L4(\% T-Score) & $-3,01( \pm 0,41)$ & $0,11( \pm 0,66)$ & $* \mathrm{p}=0,001$ \\
$\quad$ Colo Femoral (\% T-score) & $-1,81( \pm 0,67)$ & $1,10( \pm 1,97)$ & $* \mathrm{p}<0,001$ \\
$\quad$ Fêmur Total (\% T-Score) & $-1,27( \pm 0,56)$ & $0,61( \pm 0,38)$ & \\
VITAMINA D & & & \\
$\quad$ Suficiência & $55,5 \%$ & $42,86 \%$ & \\
$\quad$ Insuficiência & $44,4 \%$ & $57,14 \%$ & \\
IPAQ & & & \\
Ativo & $44,4 \%$ & $28,6 \%$ & \\
Irregularmente ativo & $55,6 \%$ & $71,4 \%$ & \\
\hline
\end{tabular}

Teste de normalidade Shapiro-Wilk $(\mathrm{p}>0,05)$

$\mathrm{DP}=$ desvio padrão

Teste T Student. Comparação: GO x GC $\left({ }^{*} p<0,05\right)$

A análise estatística mostra a homogeneidade dos grupos. Foi utilizado o teste de normalidade Shapiro-Wilk, considerando $\mathrm{p}>0,05$, sendo todos os dados paramétricos descritos como média e desvio-padrão.

Para as comparações entre os grupos, foi utilizado o Teste T Student com valor de significância $\mathrm{p}<0,05$.

\subsection{Local do Estudo}

O estudo foi realizado no Instituto de Ortopedia e Traumatologia do Hospital das Clínicas da Faculdade de Medicina da Universidade de São Paulo (IOT-HC/FMSUP), no 
Laboratório do Estudo do Movimento (LEM) e na Divisão de Radiologia em conjunto com o Departamento de Ginecologia e Obstetrícia-HC/FMUSP.

\subsection{Materiais}

- Balança mecânica e Estadiômetro (Marca Welmy®)

- Biodex ${ }^{\circledR}$ Stystem 3 modelo Biodex Multi Joint System, BIODEX SYSTEM INC., Software versão 4.5 .

- Aparelho de RNM marca General Eletrics (GE), modelo HDxt 1,5 Tesla, bobina 8-CH Body Full HD.

- Aparelho de Densitometria Óssea marca General Eletrics (GE), modelo DPX - Lunar Dual Energy (DEXA) Absorptometry.

- Equipamento LIAISON® 25 OH Vitamina D (DiaSorin Inc, MN, EUA)

- Lactímetro Accutrend® Lactate Roche.

- Tiras reagentes para lactato BM-Lactate Roche®

\subsection{Procedimentos}

As voluntárias com osteoporose (GO) foram selecionadas no ambulatório de Ginecologia Endocrinologia e Climatério do Instituto Central do Hospital das Clínicas da Faculdade de Medicina da Universidade de São Paulo e avaliadas quanto aos critérios de inclusão.

As voluntárias do Grupo-Controle (GC) foram entrevistadas por telefone e avaliadas no dia da coleta quanto aos critérios de inclusão. Todas voluntárias, que concordaram em participar, assinaram o Termo de Consentimento Livre e de Esclarecimento (TCLE).

O fluxograma dos procedimentos é visto na Figura 1. 
Figura 1. Fluxograma da avaliação e coleta de dados

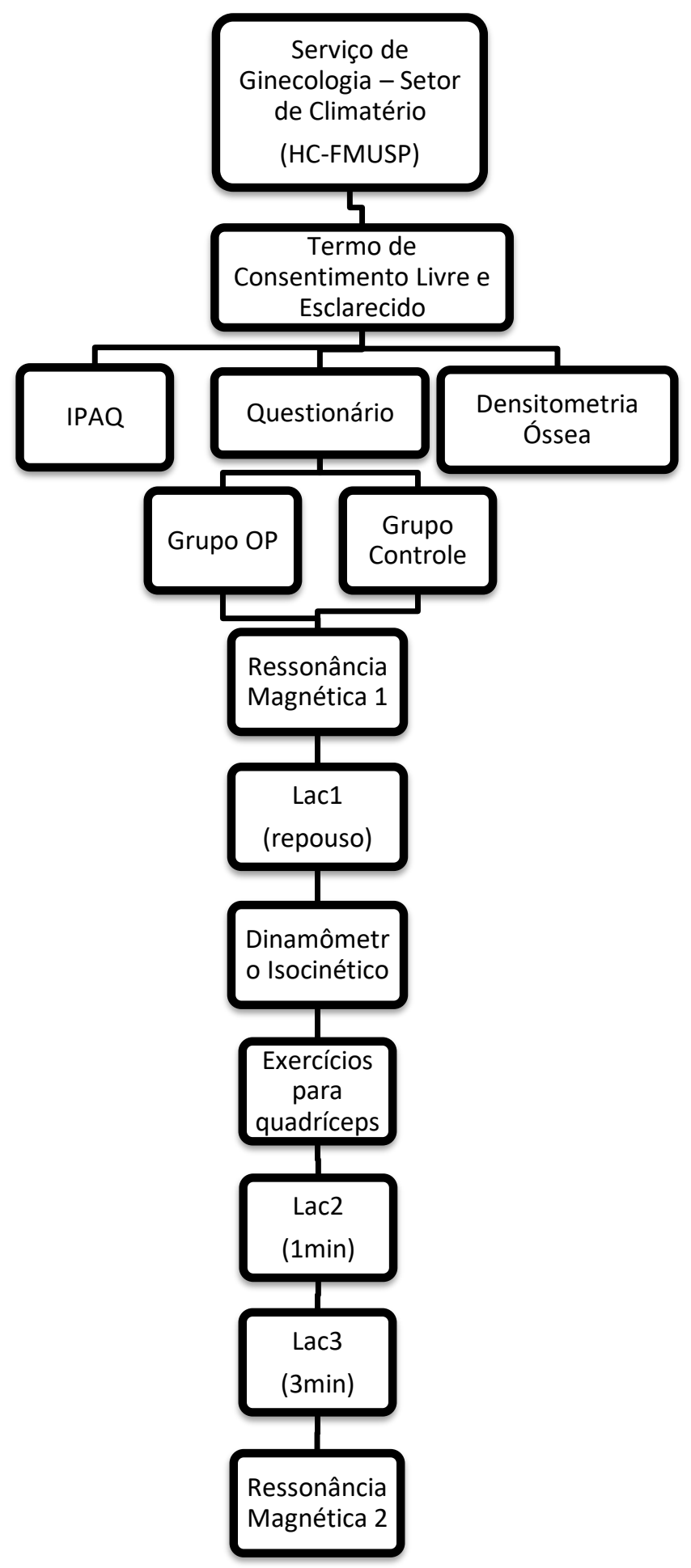




\subsection{Coleta de Dados}

\subsubsection{Questionário}

As voluntárias responderam a um questionário para inclusão e coleta de dados (Anexo 1) com as seguintes infomrções:

1. Identificação (dados demográficos);

2. História clínica pregressa de lesões (exame clínico, medicamentos e hábitos);

3. Atividade física;

4. História pregressa da Menopausa;

5. Dominância de Membro Inferior

6. Todos os indivíduos foram inicialmente submetidos ao um exame antropométrico

a) Massa Corporal (kg): utilizada uma balança mecânica com precisão de 100 gramas. O indivíduo trajando somente roupas leves (shorts e camiseta) e descalço, de frente para o avaliador e de costas para o visor da balança.

b) Estatura $(\mathrm{cm})$ : a medida realizada considerando-se a distância entre a plataforma do estadiômetro e o vértex da cabeça, tendo como base o plano de Frankfurt. O indivíduo realizou uma inspiração, seguida de um bloqueio respiratório para que a medida seja tomada.

c) Índice de Massa Corpórea (IMC): O IMC foi calculado pela equação para cálculo do IMC $=$ peso /altura ${ }^{2}$.

\subsubsection{Densitometria Óssea}

As voluntárias realizaram uma densitometria óssea. Foram aceitos exames anteriores realizados há 12 ou menos meses para determinar o grupo de inclusão pela DMO.

\subsubsection{Vitamina D e Cálcio}

Todas as voluntárias realizaram, no momento da avaliação, exame de sangue para dosagem de $25(\mathrm{OH})$ D e cálcio, que foram usados para estratificar a amostra em relação a estes parâmetros. 


\subsubsection{Protocolo de Fadiga Músculo Quadríceps Femoral}

O músculo quadríceps foi submetido a um protocolo de exercícios, com o objetivo de causar fadiga muscular, para posteriormente as pacientes serem submetidas à RNM.

\subsubsection{Dinamometria Isocinética dos Flexores/ Extensores Joelho}

Foi feita no dinamômetro isocinético modelo Biodex® Stystem 3. Foram realizadas duas séries de 10 repetições, com um minuto de intervalo entre cada série, na velocidade de $180^{\circ}$ / segundo. A frequência cardíaca e a pressão arterial foram controladas antes e após os testes. Este protocolo se baseou na literatura, que recomenda exercícios com velocidades angulares e número de repetições maiores e na avaliação funcional clínica das voluntárias (Bottaro;Russo;Oliveira, 2005; Maffiuletti et al., 2007; Brech et al., 2013, Cramer, 2015).

Figura 2. Dinamômetro isocinético

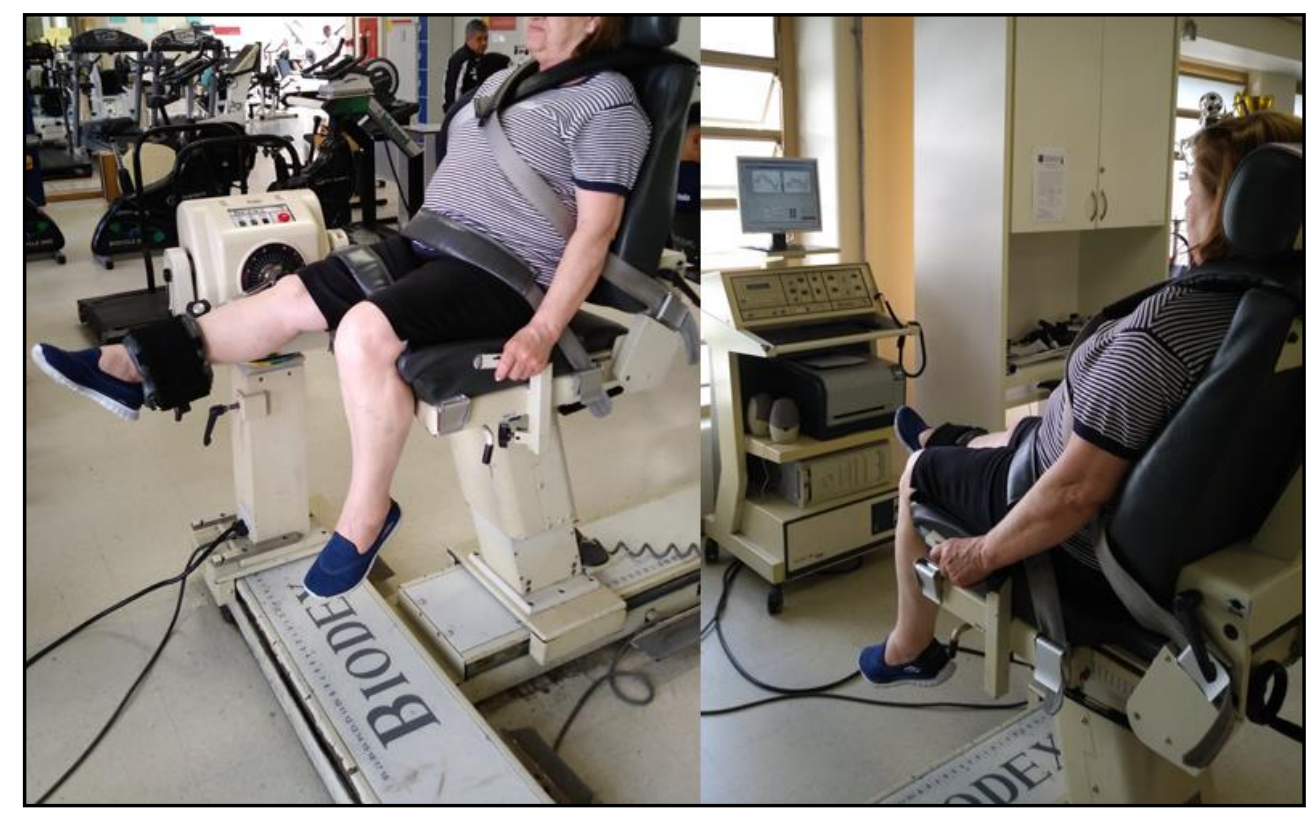

\subsubsection{Exercícios complementares}

Além da dinamometria isocinética, para manter a fadiga muscular, já no local da RNM, as voluntárias realizaram o exercício de subir e descer um degrau de $10 \mathrm{~cm}$ de altura durante cinco minutos e fizeram três agachamentos, mantendo a parte posterior do tronco apoiado na parede e a contração do quadríceps por um minuto ou até referirem fadiga. Imediatamente, após 
estas atividades, forma coletadas amostras de sangue para dosagem de lactato e as pacientes foram submetidas ao segundo exame de RNM.

Figura 3. Exercício de degrau e exercício de agachamento.

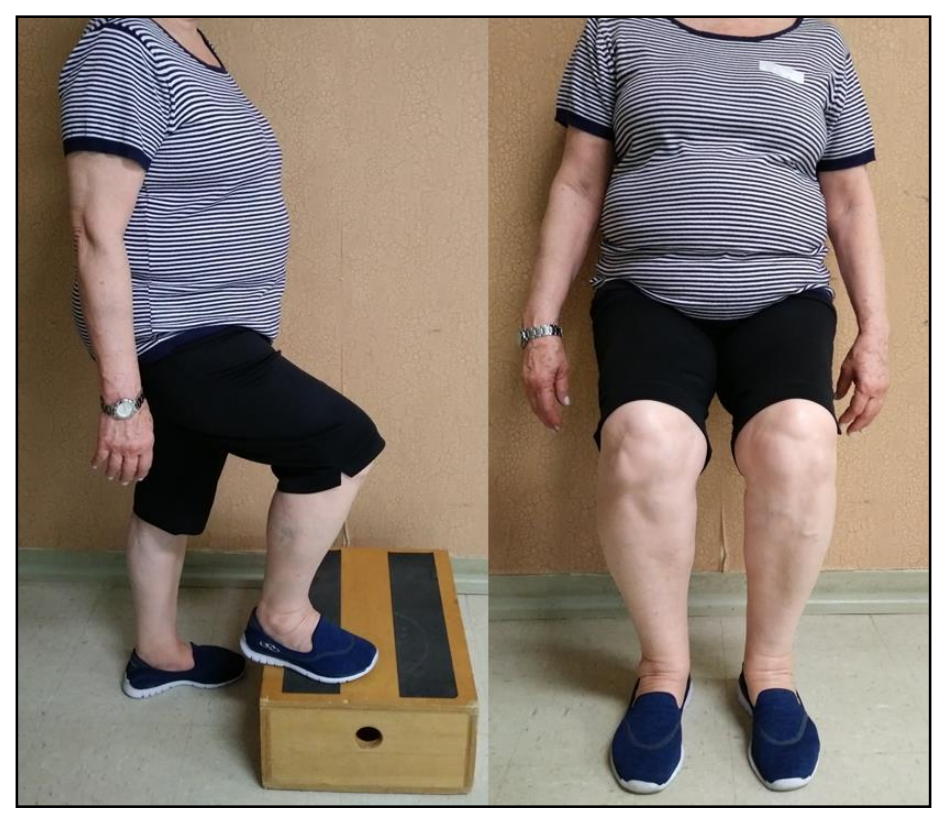

\subsubsection{Dosagem Lactato}

O lactato sanguíneo foi dosado no Lactímetro Accutrend® Lactate Roche, para comprovar a presença de fadiga muscular. Não foi na comparação com os demais resultados. Foi coletada uma gota de sangue da falange distal do terceiro ou quarto dedo, com um lancetador (Softclix $\left.{ }^{\circledR}\right)$ (regulagem 6 e microlancetas descartáveis). A gota de sangue foi colocada na zona de teste da tira reagente conectada ao aparelho (Lima et al, 2004; Greenwood et al, 2008; Baldari et al, 2009; Baker et al., 2012). Foi feita em três momentos: em repouso (Lac1) e antes da dinamometria (lactato basal), um minuto (Lac2) e três minutos (Lac3) após o término do protocolo de fadiga do músculo quadríceps.

\subsubsection{Ressonância Nuclear Magnética (RNM)}

As voluntárias foram submetidas a dois exames de RNM da região da coxa direita e esquerda, antes e depois do protocolo de fadiga do músculo quadríceps.

Previamente, à RNM foram fixados os marcadores/guias de vitamina E nas porções mediais anterior e posterior das coxas das voluntárias para garantir o posicionamento similar de cada imagem nos dois exames de RNM. 
Figura 4. Fixação dos marcadores para referência da Ressonância Magnética

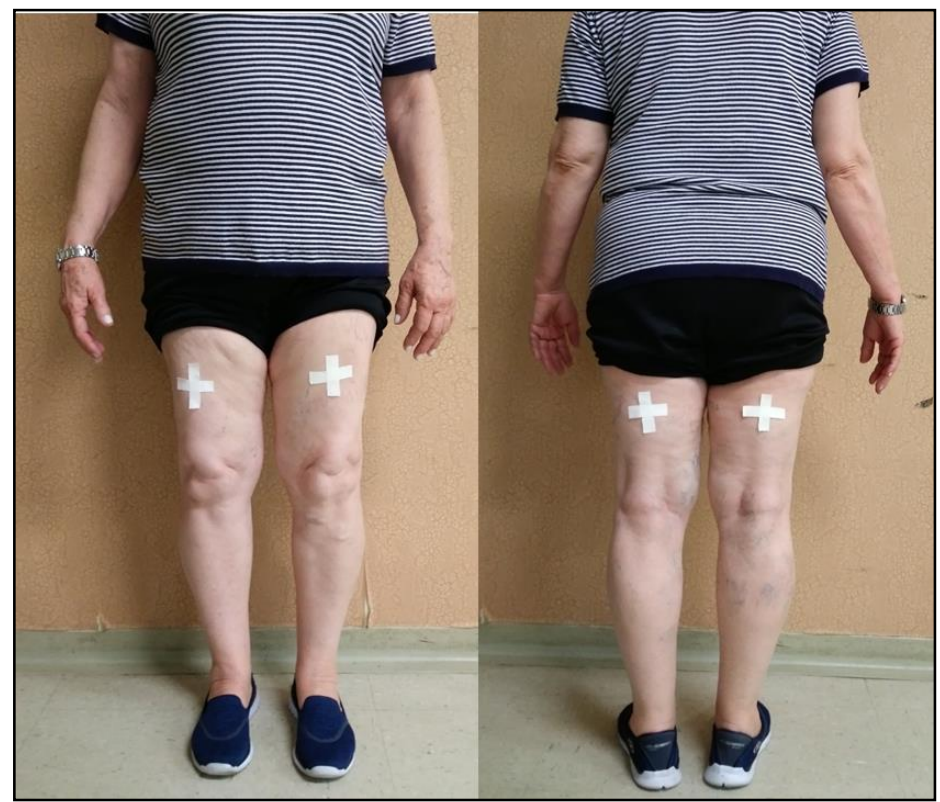

Após a colocação dos marcadores, as voluntárias foram posicionadas em decúbito dorsal na mesa deslizante acoplada ao aparelho de RNM (Aparelho General Eletrics modelo HDxt 1,5 Tesla) com a região coxofemoral bilateral posicionada dentro da bobina 8-CH Body Full HD.

Os seguintes parâmetros foram usados para as avaliações pré e pós protocolo de fadiga:

- T2 Map (FOV 38; espessura/espaçamento de corte 8.0 mm/ 1.0 mm; Matriz 256x 256; NEX 1.0; TR 1750; Multi -TE 8.0; BDW 41;24 cortes);

- Axial STIR (FOV 38; espessura/espaçamento de corte $8.0 \mathrm{~mm} / 1.0 \mathrm{~mm}$; matriz 320x 256; NEX 2.0; TR 4525; TE17; BW 31;

- Axial T1 (FOV 38; espessura/espaçamento de corte $8.0 \mathrm{~mm} / 1.0 \mathrm{~mm}$; matriz 352x 256; NEX 2.0; TR 767; TE mínimo; BW 41; 24 cortes);

- Coronal T1 (FOV 48; espessura/espaçamento de corte $5.0 \mathrm{~mm} / 1.0 \mathrm{~mm}$; matriz 384 x 256; NEX 1.0; TR 667; TE mínimo; BDW 62; 24 cortes).

O processamento das imagens foi realizado para cada sequência do mapeamento T2 em um computador ADW 4.2 GE.

Os marcadores foram localizados pelos marcadores colocados nas coxas das pacientes. A zona de interesse (bilateral) do ventre muscular do quadríceps (ROI) foi selecionada e os ROIs foram desenhados, excluindo-se pele, tecido subcutâneo, osso, vasos sanguíneos e gordura pela sequência STIR (Azzabou;Hogrel;Carlier, 2015; Azzabou; Sousa, 2015).

Foram realizados dois exames: 
RNM pré-exercício - com repouso prévio de 30 ou mais minutos para comparação do mapa T2, antes da realização do protocolo de fadiga do músculo quadríceps.

RNM pós-exercício - realizado imediatamente após o protocolo de fadiga do músculo quadríceps. O edema inflamatório, induzido pelo protocolo de fadiga do músculo quadríceps, foi avaliado no mapeamento T2, em comparação com o primeiro exame.

Figura 5. Exame de Ressonância Magnética

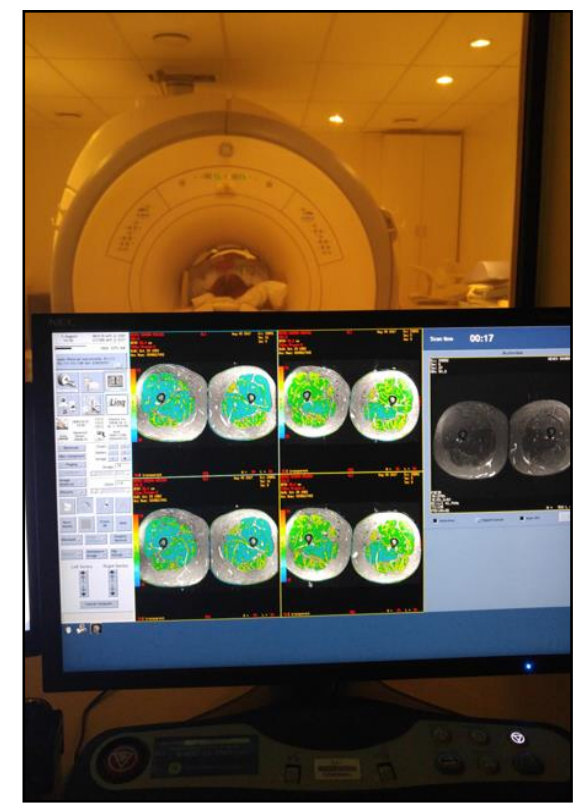

\subsection{Análise Estatística}

Os dados foram armazenados e analisados no programa IBM SPSS Statistics 22. Foi realizada a análise descritiva dos dados por meio de tabelas de frequência para as variáveis qualitativas e medidas de posição e dispersão para as quantitativas.

A normalidade e homogeneidade da variância dos dados foram confirmadas pelo teste de Shapiro-Wilk. Os dados foram reportados através de média e desvio padrão.

Foi utilizado o teste T-Student para analisar as diferenças entre as condições pré e pósexercício para as variáveis dependentes:

- RNM pré-exercício e RNM pós-exercício (medidas pré e pós-intervenção para imagem T2);

- Isocinético - Pico de torque corrigido pelo peso corporal e Trabalho total (GO: coxa D x coxa E e GC: coxa D x coxa E);

Para as variáveis independentes:

- RNM pós-exercício (GO x GC), 
- Vitamina D (GO x GC),

- Isocinético - Pico de torque corrigido pelo peso corporal e Trabalho total (GO x GC)

Para as correlações entre os resultados da RNM pós-exercício e o Pico de torque corrigido pelo peso corporal e a Vitamina D, foi utilizado Teste de Correlação de Pearson já entre a RNM pós-exercício e Trabalho total (Isocinético) foi realizado o Teste de Correlação de Spearman.

Um índice de significância de 5\% foi adotado. 


\section{RESULTADOS}

$\mathrm{Na}$ comparação intragrupo houve aumento significante $(\mathrm{p}<0,05)$ do sinal T2 da ressonância nuclear magnética (RNM) no músculo quadríceps (Tabela 2) após a realização dos exercícios nas duas coxas e em ambos os grupos.

Na comparação intergrupos, não houve diferença, estatisticamente significante, entre os Grupos Osteoporose e Controle ( $\mathrm{p}=0,883)$, mostrando que a condição óssea não interferiu na alteração do sinal T2 da RNM (Tabela 2) após os exercícios.

Tabela 2 - Mapa T2 (Sinal do tempo de relaxamento T2) (média e desvio padrão) da Ressonância Nuclear Magnética (RNM) pré e pós exercícios do Grupo Osteoporose (GO) e Grupo Controle (GC) na coxa direita (D) e esquerda (E).

\begin{tabular}{ccccc}
\hline & & N & RNM1 & RNM2 \\
\hline \multirow{2}{*}{ GO } & Coxa D & 9 & $47,13 \pm 3,04^{*}$ & $51,14 \pm 3,86^{*}$ \\
& Coxa E & 9 & $46,83 \pm 2,96^{*}$ & $51,45 \pm 3,75^{*}$ \\
\multirow{2}{*}{ GC } & Coxa D & 7 & $47,99 \pm 4,34^{\#}$ & $52,35 \pm 3,08^{\#}$ \\
& Coxa E & 7 & $46,08 \pm 3,83^{\#}$ & $51,45 \pm 2,92^{\#}$
\end{tabular}

Teste T Student. Comparação intragrupo: $\mathrm{GO}={ }^{*} \mathrm{p}<0,0001$ coxa $\mathrm{D}$ e E; $\mathrm{GC}={ }^{*} \mathrm{p}=0,002$.

Comparação intergrupo GO x GC - N.S. p >0,05 em todas comparações: pré: coxa D $\mathrm{p}=0,590$ e coxa $\mathrm{E} p=0,561$; pós: $\operatorname{coxa} \mathrm{D} \mathrm{p}=0,989$ e coxa $\mathrm{E} \mathrm{p}=0,883$

Como na comparação dos resultados da ressonância magnética entre a coxa direita e esquerda nos dois grupos estudados, não houve diferença, foi feita uma análise com os resultados das 18 coxas do GO e 14 coxas do GC, antes e depois dos exercícios. Não houve diferença, entre os grupos, no sinal T2 na RNM antes e depois da realização dos exercícios (Tabela 3). A ativação do sinal T2 em ambos grupos aumenta após os exercícios, mas não há diferença na ativação entre o GO e GC. 
Tabela 3 - Mapa T2 (Sinal do tempo de relaxamento T2) (média e desvio padrão) da Ressonância Nuclear Magnética (RNM) pré e pós-exercício do Grupo Osteoporose (GO) e Grupo Controle (GC) em ambas as coxas.

\begin{tabular}{llccc}
\hline & N & $\begin{array}{c}\text { RNM pré } \\
\text { (média e DP) }\end{array}$ & $\begin{array}{c}\text { RNM pós } \\
(\text { média e DP) }\end{array}$ & p \\
\hline GO & 18 & $46,98 \pm 2,91^{*}$ & $51,29 \pm 3,70^{*}$ & $\mathrm{p}<0,0001$ \\
GC & 14 & $47,04 \pm 4,06^{\#}$ & $51,90 \pm 2,92^{\#}$ & $\mathrm{p}<0,0001$ \\
\hline
\end{tabular}

T Student. Comparação intergrupos GO x GC: pré-exercício p=0,343 e pós-exercício

$$
\mathrm{p}=0,874
$$

Figura 6. RNM 1 - Imagem Ressonância Magnética em repouso. RNM 2 - Imagem Ressonância Magnética pós-exercício.

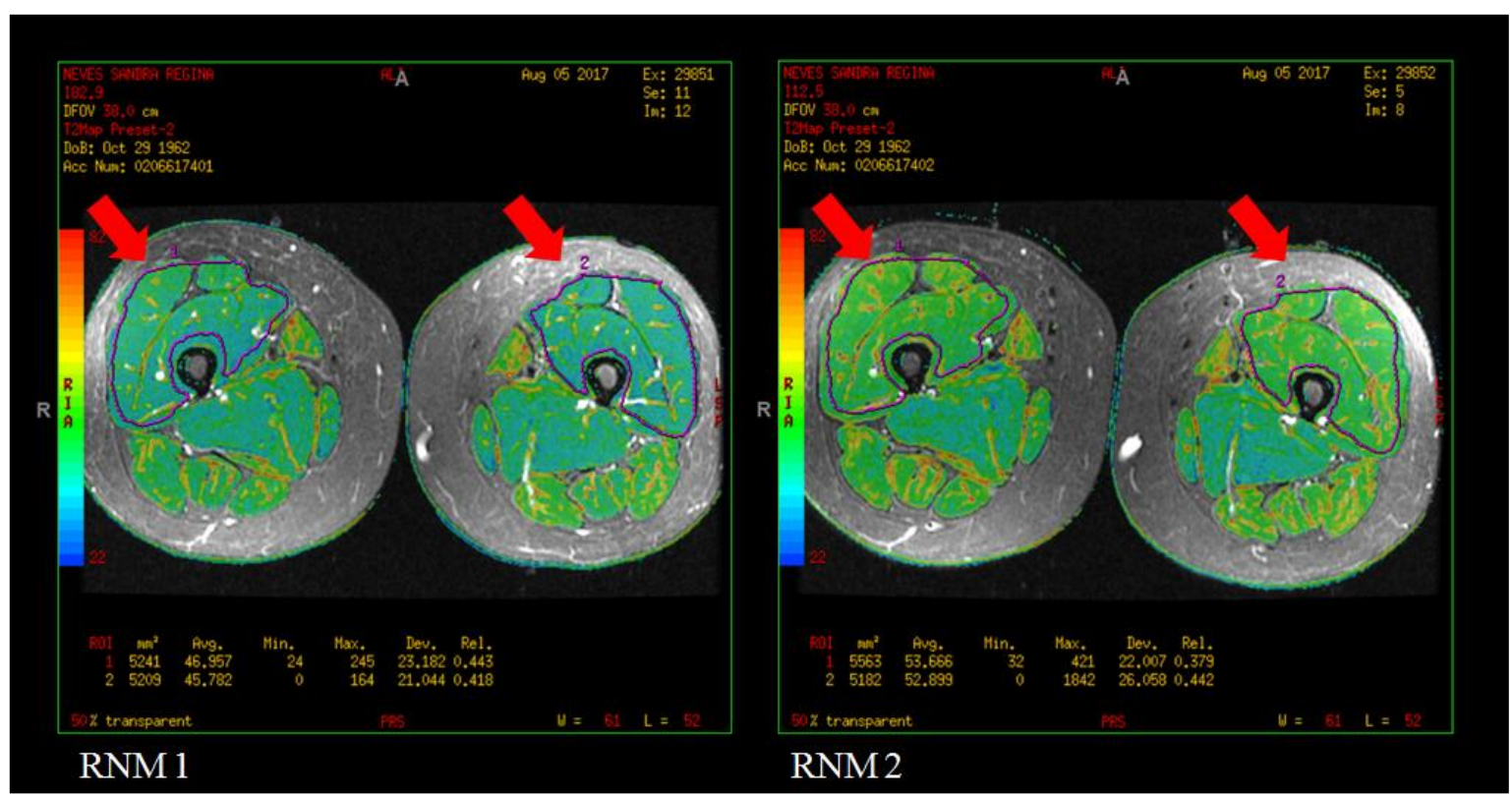

Tabela 4 - Variação dos níveis sanguíneos de Lactato

\begin{tabular}{cccc}
\hline & GO & GC & p \\
\hline Lac1 & $2,90( \pm 0,59)$ & $2,38 \pm(0,71)$ & $\mathrm{p}=0,742$ \\
Lac2 & $5,52 \pm(1,16)$ & $3,87 \pm(1,07)$ & $* \mathrm{p}=0,018$ \\
Lac3 & $6,31 \pm(0,76)$ & $4,35 \pm(1,15)$ & $* \mathrm{p}=0,002$
\end{tabular}

Lac1 = índice em repouso; Lac2 = índice após 1 minuto da execução dos exercícios; Lac3 = índice após 3 minuto da execução dos exercícios.

$\mathrm{DP}=$ desvio padrão. Teste T Student. Comparação: GO x GC $\left({ }^{*} \mathrm{p}<0,05\right)$ 
Os resultados mostram que houve uma variação significante comparação entre o Lac1 (lactato de repouso) com o Lac3 (lactato após 3 minutos da execução dos exercícios) em ambos os grupos, GO $(\mathrm{P}<0.0001)$ e GC $(\mathrm{P}<0.0001)$ (Tabela 4).

\section{Correlações da Ressonância Nuclear Magnética pós-exercícios (RNM2)}

Os dados da dinamometria isocinética (Tabela 5) o pico de torque corrigido pelo peso corporal (força muscular) e trabalho total de todas as repetições (potência), com valor significante quando comparada coxa direita e coxa esquerda.

Na comparação dos resultados da vitamina D entre os grupos GO e GC, não houve diferença entre os grupos (Tabela 6)

Como não houve diferença significante nos valores da dinamometria isocinética entre membro inferior direito e esquerdo, as correlações foram feitas com os resultados da RNM pósexercícios com pico de torque corrigido pelo peso corporal e a potência das duas pernas, foi realizada ainda correlação entre RNM e valores da dinametria isocinética com a vitamina D (Tabela 7).

Tabela 5 - Dinamometria isocinética (média e desvio padrão) de mulheres pós-menopáusicas com e sem osteoporose.

\begin{tabular}{|c|c|c|c|c|}
\hline & $\mathbf{N}$ & Coxa D & Coxa $E$ & $\mathbf{p}$ \\
\hline \multicolumn{5}{|c|}{ Pico De Torque/Peso Corporal (\%) } \\
\hline GO & 9 & $93,14 \pm 10,23 *$ & $95,70 \pm 16,59 *$ & $\mathrm{p}<0,001$ \\
\hline GC & 7 & $82,62 \pm 23,43 *$ & $81,05 \pm 25,44^{*}$ & $\mathrm{p}<0,001$ \\
\hline \multicolumn{5}{|c|}{ Trabalho Total (W) } \\
\hline GO & 9 & $539,7 \pm 122,0^{\#}$ & $548,2 \pm 98,3^{\#}$ & $\mathrm{p}<0,001$ \\
\hline GC & 7 & $623,6 \pm 156,2^{\#}$ & $601,4 \pm 191,8^{\#}$ & $\mathrm{p}<0,001$ \\
\hline
\end{tabular}

Teste T Student. Comparação intergrupos: Pico de torque corrigido pelo peso corporal GO x GC coxa $D p=0,416$ e coxa $E \mathrm{p}=0,313$. Trabalho Total $\operatorname{coxa} \mathrm{D} p=0,157$ e coxa $E \mathrm{p}=0,334$. 
Tabela 6 - Dosagem de 25 OH Vitamina D (ng/ml) (média e desvio padrão) em mulheres pósmenopáusicas com e sem osteoporose

\begin{tabular}{llll}
\hline & N & 25 OH Vitamina D & p \\
\hline GO & 9 & $29,50 \pm 5,83$ & $\mathrm{p}=0,927$ \\
GC & 7 & $29,08 \pm 10,32$ &
\end{tabular}

Teste T Student.

Tabela 7 - Distribuição dos valores de coeficiente de correlação e valor de p referentes ao trabalho do músculo quadríceps entre os valores do Dinamômetro Isocinético, Ressonância Nuclear Magnética e Vitamina D

\begin{tabular}{lcccc}
\hline & & RNM2 & 25 OH Vitamina \\
& & & (mapa T2) & D (ng/ml) \\
\hline Pico de & Torque/Peso & $\mathrm{r}$ & $-0,076$ & $-0,316$ \\
Corporal (\%) & $\mathrm{p}$ & 0,846 & 0,233 \\
\hline \multirow{2}{*}{ Trabalho Total (w) } & $\mathrm{r}$ & 0,276 & 0,329 \\
& $\mathrm{p}$ & 0,139 & 0,066 \\
\hline \multirow{2}{*}{ RNM2 } & $\mathrm{r}$ & - & $-0,171$ \\
& $\mathrm{p}$ & - & 0,525 \\
\hline
\end{tabular}

Correlação de Pearson para as variáveis Pico de Torque/Peso Corporal (\%), RNM2 e 25 OH Vitamina D e Correlação de Spearman para a variável Trabalho Total (w). 


\section{DISCUSSÃO}

Este estudo mostra que a RNM por meio do mapa T2 é um método eficaz para mostrar as alterações musculares agudas após exercícios.

As mulheres com e sem osteoporose apresentaram o mesmo padrão de ativação muscular, mostrando que na faixa etária estudada, a osteoporose não influenciou o metabolismo muscular. Não foram encontrados na literatura estudos que avaliassem a alteração metabólica muscular pelo mapa T2 em mulheres com e sem osteoporose, no período pós-menopausa com RNM, para comparação.

Após a realização de uma atividade física, é gerado o lactato como consequência da glicólise anaeróbica poduzida por todos os tecido do corpo, sendo este um marcado para índice de fadiga pós-exercício (Di Mauro; Scoeffler, 2016). Deste modo, Podemos observer que o protocolo de exercícios utilizados no atual estudo causou fadiga na musculatura extensora do joelho, pois houve um aumento do ácido lático nas duas medidas após a realização dos exercícios, tal achado corrobora com Baker et al., 2012 que mensurarm as variações do lactato sanguineo utilizando o mesmo método da presente pesquisa, após 10 e 20 segundos de atividade máxima no cicloergômetro e observaram diferença com aumento na dosagem deste marcador sanguíneo.

Participaram do estudo 16 mulheres com idade entre 55 e 65 anos na fase pósmenopausa, que foram separadas pelo T-Score da densidade mineral óssea (DMO) nos grupos Osteoporose e Controle. Todas participantes eram ativas ou irregularmente ativas pelo IPAQ, instrumento confiável e validado para a língua portuguesa (Matsudo et al., 2002). O IPAQ questiona as atividades físicas realizadas na última semana, mas não traz informações sobre frequência e intensidade da atividade física realizada, ainda sim é um bom método de estratificação da amostra. De qualquer maneira, nenhuma das participantes estudadas fazia atividade física ou esporte de forma regular, fato que contribuiu para a homogeneidade da amostra estudada. Contudo, Azzabou, Hogrel e Carlier (2015) relatam que a idade é um fator importante para as alterações captadas da RNM muscular, porém não encontraram correlação destas alterações com nível de atividade física.

A prevalência da sarcopenia varia pelas diferentes definições e métodos de mensuração da composição corporal (Cruz-Jentoft et al, 2010; Cruz-Jentoft;Landi, 2014; Go et al, 2013). Tarantino et al., 2013 destacam a sarcopenia e a osteoporose como alterações morfológicas importantes decorrentes da idade. Porém, o envelhecimento parece estar diretamente 
relacionado com diminuição progressiva de massa muscular, com uma associação significante entre sarcopenia e osteoporose, mais prevalente em mulheres. (Di Monaco et al, 2011; Drey et al., 2015; Kawao;Kaji, 2015), fato que embasa a hipótese inicial do atual estudo: se as mulheres com osteoporose poderiam ter menor ativação muscular após realização dos exercícios, pela possível presença de sarcopenia associada. Em contraponto, os resultados desta pesquisa não confirmaram tal hipótese, uma vez que quando comparados os resultados do GO e GC os grupos mostraram comportamentos semelhantes em relação ao mapa T2 pré e pós a realização dos exercícios, sem diferença estatística entre eles.

A RNM aparece como ferramenta importante e não invasiva para avaliação muscular (Martins-Bach et al., 2015; Mendiguchia et al., 2013) por meio da captação das alterações da atividade metabólica das células musculares pelo aumento da intensidade do sinal T2 (Azzabou;Sousa., 2015).

O mapa T2 é uma ferramenta sensível para avaliar as alterações metabólicas agudas do músculo após uma sessão de exercícios, sendo neste estudo observado aumento do sinal do mapa T2 após a série de exercícios realizada para o músculo quadríceps, fato que confirma que ocorreu aumento significativo da atividade metabólica das células musculares, ainda que como já mencionado, sem diferença entre os grupos avaliados, o trabalho de Mendiguchia et al. (2013) estudaram os músculos posteriores da coxa de jogadores de futebol e confirmam a eficácia da RNM como método de captação das alterações musculares agudas pós treinamento pelo aumento do sinal de T2, assim como Da Cruz (2014) que relata maior atividade metabólica dos músculos reto-femoral, vasto medial e glúteo máximo usando o mapa T2 da RNM imediatamente após treinamento com o Método Pilates.

A literatura mostra uma forte correlação entre as alterações musculares e ósseas (Di Monaco et al, 2011; Tarantino et al., 2013, Drey et al., 2015; Kawao;Kaji, 2015), em relação à resposta aos exercícios nas mulheres com osteoporose na faixa etária estudada. Em nossos resultados, porém, não foi encontrada diferença na ativação muscular das mulheres com e sem osteoporose, sinalizando que não há uma associação obrigatória da osteoporose com sarcopenia. Baseados nestes achados, podemos afirmar que a condição muscular das mulheres no período pós menopausa com e sem osteoporose, em relação ao padrão de ativação metabólica dado pelo mapa T2 não é diferente, visto que a resposta muscular aos exercícios foi a mesma nos dois grupos. Tarantino et al., (2013) sugerem que a interação osso-músculo é fundamental para o desenvolvimento de novos instrumentos de diagnóstico e novas terapias para disfuções musculo-esqueléticas. 
Nossos resultados podem ser explicados, pelo menos parcialmente, pela amostra estudada, mulheres relativamente mais jovens, ativas, saudáveis e ainda sem as complicações mais graves da osteoporose, mas também mostram de forma inequívoca que a resposta muscular ao exercício é a mesma nos dois grupos. Este achado sugere, fortemente, que nesta fase mulheres no período pós-menopausa podem ser submetidas a programas de exercícios e apresentarem resposta positiva, apesar de sua condição óssea.

Os resultados encontrados desmentem a hipótese inicial do estudo, mas são promissores em relação à indicação dos exercícios para mulheres com osteoporose, pois pode se esperar uma boa resposta muscular aos mesmos, fato que pode ajudar na prevenção da sarcopenia e osteoporose, além de ser importante também na prevenção da fragilidade e suas consequências como as quedas e fraturas, fatores importantes de impacto na saúde de mulheres pósmenopáusicas (Fried et al. 2001; Brincat et al., 2015).

Brech et al. (2013) referem diminuição da força muscular dos extensores e flexores do joelho e equilíbrio nas mulheres com osteoporose, em estudo com 126 mulheres na fase pósmenopausa, dado não observado no estudo atual, visto que o pico de torque extensor (músculo quadríceps), medida de força muscular, obtido pela dinamometria isocinética, foi o mesmo nos dois grupos avaliados, fato que pode ser justificado pelo tamanho da amostra, mas também pela homogeneidade da mesma em relação à atividade física e idade. Foi feita, então, a correlação entre o pico de torque (força muscular) e a intensidade do sinal do tempo de relaxamento T2, em todas as participantes, juntando-se os dois grupos, ainda assim se observou correlação significante. A falta de correlação pode ter sido dada pelo tamanho amostral, mas também pela grande variabilidade dos valores do pico de torque.

Apesar de estar descrito na literatura que a deficiência da vitamina D causa hipotrofia das fibras tipo II (Glerup et al., 2000; Phu;Derek;Duque, 2015; Tarantino et al., 2013; Brincat et al., 2015; Wu et al., 2016) pela sua ação moduladora no controle dos hormônios que atuam sobre os ossos e músculos (Gunton et al. 2015; Girgis et al. 2015) de nenhuma participante, de acordo com os critérios de inclusão, realizar a suplementação de vitamina $\mathrm{D}$, no momento da avaliação, ação que pode reduzir os índices de perda óssea e de força muscular (Bączyk et al., 2012; Maghraoui et al., 2012, Brincat et al., 2015, Drey et al., 2015; Kühn;Trotz;Stangl, 2018), não foram encontrados resultados significantes quando feitas as correlações da dosagem de vitamina $\mathrm{D}$, detectada previamente por exame de sangue, com o mapa T2 e com as variáveis de força e potência da dinamometria isocinética, porém o tamanho amostral é uma limitação para análise segura destes achados. 
A falta de correlações significantes pode ser explicada pela faixa etária escolhida para compor a amostra (55 a 65 anos), que apesar de poder ter funcionado como uma limitação do estudo, tratou-se de escolha proposital, para evitar a influência de maiores declínios decorrentes do processo de envelhecimento nas condições musculares.

Outra limitação que restringiu o tamanho amostral foi a disponibilidade da ressonância nuclear magnética para a pesquisa, pois as prioridades de uso são para a assistência aos pacientes.

Também é reconhecido que a avaliação da composição corporal pela densitometria (DEXA) poderia ajudar no entendimento e quantificação de tecidos com e sem gordura. 


\section{CONCLUSÃO}

A ativação muscular aguda do quadríceps avaliado pelo Mapa T2 da RNM é igual nas mulheres com e sem osteoporose na fase pós-menopausa e pode se afirmar que a densidade mineral óssea não interfere na resposta muscular aos exercícios.

A dosagem de vitamina $\mathrm{D}$ não interferiu na força e na ativação muscular, mas o pequeno tamanho amostral é uma ressalva à esta afirmativa. 


\section{BIBLIOGRAFIA}

Aidelsburger P, Schauer S, Grabein K, Wasem J. Alternative methods for the treatment of postmenopausal troubles. GMS Health Technology Assessment 2012;8:1-9.

Aquino MA, Leme LEG. Isokinetic dynamometry in elderly women undergoing total knee arthroplasty: a comparative study. Clinics. 2006;61(3):215-22.

Arantes HP, Gimeno SFA, Chiang AY, Bilezikian JP, Lazaretti-Castro M. Incidence of vertebral fractures in calcium and vitamin D-supplemented postmenopausal Brazilian women with osteopenia or osteoporosis: data from Arzoxifene Generations Trial. Arch Endocrinol Metab. 2016;60/1:54-59.

Azzabou N, Hogel JY, CArlier PG. NMR based biomarkers to study age-related changes in the human quadríceps. Experimental Gerontology. 2015;70:54-60.

Azzabou N, Sousa PL. Validation of a Generic Approach to Muscle Water T2 Determination at 3T in Fat-Infiltrated Skeletal Muscle. J. Magn. Reson. Imaging 2015;41:645-653.

Bączyk G, Opala T, Kleka P, Chuchracki M. Multifactorial analysis of risk factors for reduced bone mineral density among postmenopausal women. Arch Med Sci 2012; 8(2): 332-41.

Baker JS, Thomas N, Cooper SM, Davies B, Robergs RA. Exercise duration and blood lactate concentrations following high intensity cycle ergometry. Res Sports Med. 2012; 20(2):129-4.

Baldari C, Bonavolontà V, Emerenziani GP, Galotta MC, Siva AJ, Guidetti L. Accuracy, reliability, linearity of Accutrend and Lactate Pro versus EBIO plus analyzer. Eur J Appl Physio. 2009; 107 (1):105-11.

Bandeira F, Griz L, Freese E, Lima DC, Thé AC, Diniz ET, Marques TF, Lucena CS. Vitamin D deficiency and its relationship with bone mineral density among postmenopausal women living in the tropics. Arq Bras Endocrinol Metab. 2010;54(2):227-32. 
Bottaro M, Russo A, Oliveira RJ. The effects of rest interval on quadriceps torque during an isokinetic testing protocol in elderly. J Sports Sci Med 2005; 4:285-290.

Brasil. Ministério da Saúde. Portal da Saúde. Vigitel Brasil 2010: vigilância de fatores de risco e proteção para doenças crônicas por inquérito telefônico. Brasília: Ministério da Saúde, 2011. Disponível em: http://portal.saude.gov.br/portal/arquivos/pdf/vigitel_180411.pdf. Acessdo em 03 de agosto 2017.

Brech GC, Plapler PG, Meirelles ES, Marcolino FMD, Greve JMD. Evaluation of the association between osteoporosis and postural balance in postmenopausal women. Gait \& Posture. 2013; 38:321-325.

Brincat M, Gambin J, Brincat M, Calleja-Agius J. The role of vitamin D in osteoporosis. Maturitas. 2015;80:329-332.

Carvalho M, Kurlak CAM, Borba VZC. Prevalência de hipercalciúria em mulheres na pósmenopausa com osteoporose. Arq Bras Endocrinol Metab. 2012; 56(1):1-5.

Cramer JT, Jenkins ND, Mustad VA, Weir JP. Isokinetic Dynamometry in Healthy Versus Sarcopenic and Malnourished Elderly: Beyond Simple Measurements of Muscle Strength. $J$ Appl Gerontol, 2015.

Cranney A, Weiler HA, O’Donnell S, Puil L. Summary of evidence-based review on vitamin D efficacy and safety in relation to bone health. Am J Clin Nutr. 2008;88(suppl):513S-9S.

Cruz-Jentoft AJ e Landi F. Sarcopenia. Clinical Medicine. 2014;14(2):183-6.

Cruz-Jentoft AJ, Baeyens JP, Bauer JM, Boirie Y, Cederholm T, Landi F, Martin FC, Michel JP, Rolland Y, Schneider SM, Topinková E, Vandewoude M, Zamboni M. Sarcopenia: European consensus on definition and diagnosis. Age Agening 2010; 39(4):412-423.

Da Cruz TMF. Alterações morfológicas, funcionais e fisiológicas de uma sessão de pilates com aparelhos. Piracicaba: Universidade Metodista de Piracicaba; 2014. 
Di Monaco M, Vallero F, Di Monaco R, Tappero R. Prevalence of sarcopenia and its association with osteoporosis in 313 older women following a hip fracture. Arch of Gerontology and Geriatrics; 2011; 52:71-74.

Di Mauro FM, Scoeffler GL. Point of Care Measurement of Lactate. Top Companion Anim Med. 2016 Mar;31(1):35-43.

Doubova SV, Alarcón PE, Hernández SF, Infante C, Cuevas RP. Integrative health care model for climacteric stage women: design of the intervention BMC Women's Health 2011, 11:6.

Drey M, Sieber CC, Bertsch T, Bauer JM, Schmidmaier R. Osteosarcopenia is more than sarcopenia and osteopenia alone. Aging Clin Exp Res. 2015.

Esposito A, Campana L, Palmisano A, Cobelli F, Canu T, et al. Magnetic Resonance Imaging at $7 \mathrm{~T}$ reveal commonevents in age-related sarcopenia and in the homeostatic response to muscle sterile injury. PLoS ONE. 2013;8(3):1-9.

Fried LP, Tangen CM, Walston J, Newman AB, et al. Frailty in Older Adults: Evidence for a Phenotype. Journal of Gerontology: Medical Sciences. 2001; 56(3):M146-M156

Girgis CM, Baldock PA, Downes M. Vitamin D, muscle and bone: Integrating effects in development, aging and injury. Molecular and Cellular Endocrinology. 2015; 410:3-10.

Glerup H, Mikkelsen K, Poulsen L, Hass E, Overbeck S, Andersen H, Charles P, Eriksen EF. Hypovitaminosis D myopathy without biochemical signs of osteomalacic bone involvement. Calcif Tissue Int. 2000;66(6):419-24.

Go SW, Cha YH, Lee JA, Park HS. Association between Sarcopenia, Bone Density, and HealthRelated Quality of Life in Korean Men. Korean J Fam Med. 2013;34:281-288.

Greenwood JD, Moses GE, Bernardino FM, Gaesser GA, Weltman A. Intensity of exercise recovery, blood lactate disappearance, and subsequent swimming performance. J Sports Sci 2008; 26(1):29-34. 
Gunton JE, Girgis CM, Baldock PA, Lips P. Bone muscle interactions and vitamin D. Bone. 2015;80:89-94.

Hellekson KL. NIH releases statement on osteoporosis prevention, diagnosis, and therapy. Am Fam Physician. 2002;66(1):161-2.

Kawao N, Kaji H. Interactions Between Muscle Tissues and Bone Metabolism. Journal of Cellular Biochemistry. 2015; 116:687-695.

Kühn J; Trotz P; Stangl GI. Prevalence of vitamin D insufficiency and evidence for disease prevention in the older population. Z Gerontol Geriat. 2018.

Lima EV, Tortoza C, Rosa LCL, Lopes-Martins AB. Estudo da correlação entre a velocidade de reação motora e o lactato sanguíneo, em diferentes tempos de luta no judô. Rev Bras Med Esporte 2004;10(5):339-343.

Maffiuletti NA, Jubeau M, Munzinger U, Agosti F, De Col A, Lafortuna CL, Sartorio A. Differences in quadriceos muscle strength and fatigue between lean and obese subjects. Eur $J$ Appl Physiol 2007;101(1):51-59.

Maghraoui AE, Ouzzif Z, Mounach A, Rezqi A, Achemlal L, Bezza A, Tella S, Dehhaoui M, Ghozlani I. Hypovitaminosis D and prevalent asymptomatic vertebral fractures in Moroccan postmenopausal women. BMC Women's Health. 2012;12(1):11.

Martins-Bach AB, Malheiros J, Matot B, Martins PCM, Almeida CF, Caldeira W, et al. Quantitative T2 Combined with Texture Analysis of Nuclear Magnetic Resonance Images Identify Different Degrees of Muscle Involvement in Three Mouse Models of Muscle Dystrophy: mdx, Large myd and mdx/Large myd. PLoS ONE 10(2):(2015)

Matsudo SM, Matdsudo VR, Araújo T, Andrade D, Andrade E, Oliveira L, Braggion G. Nível de atividade física da população do Estado de São Paulo: análise de acordo com o gênero, idade, nível socioeconômico, distribuição geográfica e de conhecimento. Rev bras ciênc Mov. 2002;10(4): 41-50. 
Mendiguchia J, Garrues MA, Cronn JB, Contreras B, Los Arcos A, Malliarpoulos N, Maffulli $\mathrm{N}$, Idoate F. Nonuniform changes in MRI measurements of the thigh muscles after two hamstring strengthening exercises. Journal of Strength and Conditioning Research 2013; $27(3) / 574-581$.

Meyer RA, Prior BM. Functional magnetic resonance imaging of muscle. Exercise and Sport Sciences Reviews 2000;28(2):89-92.

Patten C, Meyer RA, Fleckenstein JL. T2 mapping of muscle. Seminars in Musculoskeletal Radiology 2003; 7(4):297-305.

Pedrosa MAC, Castro ML. Papel da vitamina D na função neuro-muscular. Arq Bras Endocrinol Metab. 2005;49(4):495-502.

Phu S, Derek B, Duque G. Exercise and Sarcopenia. J Clin Densitom. 2015;18(4):488-92.

Pimenta F, Leal I,Maroco J, Ramos C. Menopausal symptoms: Do life events predict severity of symptoms in peri- and postmenopause? Maturitas. 2012;72:324-331.

Prior BM, Foley JM, Jayaraman RC, Meyer RA. Pixel T2 distribution in functional magnetic resonance images of muscle. J Appl Physiol 1999;87:2107-2114.

Reijnierse EM, Trappenburg MC, Leter MJ, Blauw GJ et al. The Impact of Different Diagnostic Criteria on the Prevalence of Sarcopenia in Healthy Elderly Participants and Geriatric Outpatients. Gerontology. 2015;61(6):491-6.

Ribeiro MM, Rumor L, Oliveira M, O’Neil JG, Maurício JC. STIR, SPIR and SPAIR techniques in magnetic resonance of the breast: a comparative study. J Biomedical Science and Engeneering. 2013;6:395-402.

Roberts H; Hickey M. Managing the menopause: an update. Maturitas. 2016; 86:53-58.

Sapre, S, Thakur, R. Lifestyle and dietary factors determine age at natural menopause. Journal of Mid-life Health. 2014;5:3-5 
Sandhu SK, Hampson G. The pathogenesis, diagnosis, investigation and management of osteoporosis. J Clin Pathol. 2011;64:1042-1050.

Sciorati C, Esposito A, Campana L, Canu T, Monno A, Palmisano A, Cobelli F, et al. 7-Tesla Magnetic Resonance Imaging Precisely and Noninvasively Reflects Inflammation and Remodeling of the Skeletal Muscle in a Mouse Model of Antisynthetase Syndrome. BioMed Research International. 2014:1-9.

Sousa PL, Vignaud A, Araújo ECA, Carlier PG. Factors controlling T2 mapping from partially spoiled SSFP sequence: optimization for skeletal muscle characterization. Magnetic Resonance in Medicine 2012;67:1379-90.

Souza MPG. Diagnóstico e tratamento da osteoporose. Rev Bras. Ortop. 2010;45(3):220-9.

Tawara N, Nitta O, Kumura H, Niitsu M, Itoh A. T2 mapping of muscle activity using ultrafast imaging. Magn Reson Med Sci 2011; 10(2):85-91.

Tarantino U, Baldi J, Celi M, Rao C, Liuni FM, Iundusi R, Gasbarra E. Osteoporosis and sarcopenia: the connections. Aging Clin Exp Res. 2013:25(Suppl 1):S93-S95.

The North American Menopause Society (NAMS). Consensus opinion. The role of calcium in peri- and postmenopausal women: consensus opinion of The North American Menopause Society of The North American Menopause Society Menopause. The Journal of The North American Menopause Society.2001;8(2):84-95.

Velde ER, Dorland M, Broekmans FJ. Age at menopause as a marker of reproductive ageing. Maturitas. 1998;30(2):119-125.

Wakahara T, Miyamoto N, Sugisaki N, Murata K, et al. Association between regional differences in muscle activation in one session of resistance exercise and in muscle hypertrophy after resistance training. Eur J Appl Physiol. 2012 Apr;112(4):1569-76 
World Health Organisation. Assessment of fracture risk and its implication to screening for postmenopausal osteoporosis: Technical report series 843. Geneva: WHO,1994.

Wu F, Callisaya M, Laslett LL, Wills K, Zhou1 Y, Jones G, Winzenberg T. Lower limb muscle strength is associated with poor balance in middle-aged women: linear and nonlinear analyses. Osteoporos Int. 2016

Zabka FF, Valente HG, Pacheco AM. Isokinetic evaluation of knee extensor and flexor muscles in professional soccer players. Rev Bras Med Esporte 2011;17(3):189-92. 


\section{ANEXOS}

ANEXO I

\section{QUESTIONÁRIO}

DADOS PESSOAIS

Nome:

sexo: ( )

$\mathrm{M}(\mathrm{f}) \mathrm{F}$

Data de Nascimento:

Idade:

Endereço:

Cidade:

CEP:

Telefone:

Celular:

e-mail:

EXAME CLÍNICO

\begin{tabular}{|l|l|l|l|}
\hline Problemas de Saúde & Sim & Não & Observações \\
\hline Pressão Arterial Alta & & & \\
\hline Pressão Arterial Baixa & & & \\
\hline Problema Cardíaco & & & \\
\hline Triglicérides Alto & & & \\
\hline Tireóide & & & \\
\hline Diabetes & & & \\
\hline Doenças Neurológica & & & \\
\hline \multicolumn{1}{|c}{ Crises Convulsivas } & & & \\
\hline Outros & & & \\
\hline
\end{tabular}

\begin{tabular}{|l|l|l|l|}
\hline Sintomas & Sim & Não & Observações \\
\hline Dores de cabeça & & & \\
\hline Tonturas & & & \\
\hline Dor muscular & & & \\
\hline Fraqueza Muscular & & & \\
\hline Enrijecimento Articular & & & \\
\hline Dor na coluna & & & \\
\hline
\end{tabular}


Outros

\section{MEDICAMENTOS}

Usa medicamentos regularmente: ( ) sim ( ) não

Medicamentos:

\section{HÁBITOS COMUNS}

\begin{tabular}{|l|l|l|l|l|}
\hline & Sim & Não & Tipo & Quanto \\
\hline Tabagismo & & & & \\
\hline Etilismo & & & & \\
\hline Outros & & & & \\
\hline
\end{tabular}

\section{HISTÓRIA DE ATIVIDADE FÍSICA}

\section{Pratica atividade física : ( )sim ( ) Não}

Se sim:qual(s) atividade?

Há quanto tempo?

Quantas vezes por semana: quantas horas por dia:

Anteriormente já praticou alguma atividade física com regularidade (2x/semana, no mínimo?) ( ) $\operatorname{sim}$ ( )não

Se sim: qual(s) atividade?

Por quanto tempo?

\section{HISTÓRIA DA MENOPAUSA}

Com quantos anos parou de menstruar?

Há quanto tempo? 
Como foi? ( ) Natural ( ) Cirúrgico

Sente ondas de calor? ( )Sim ( ) Não

Fez ou faz tratamento de reposição hormonal? ( ) )Sim ( )Não

DOMINANCIA DE MEMBRO INFERIOR

Qual lado que a senhora chuta? ( ) D （ ) E

DADOS ANTROPOMÉTRICOS

Massa corpórea $(\mathrm{kg})$ :

Estatura $(\mathrm{cm})$ :

IMC:

$\mathrm{kg} / \mathrm{m}^{2}$

EXAME DE SANGUE Dosagem de 25 OH Vitamina D: ng/ml. NL: 9,0 a

$37,6 \mathrm{ng} / \mathrm{ml}(\mathrm{HC})$.

DENSITOMETRIA ÓSSEA: Data: 
ANEXO II - QUESTIONÁRIO PRÉ-EXAME RM

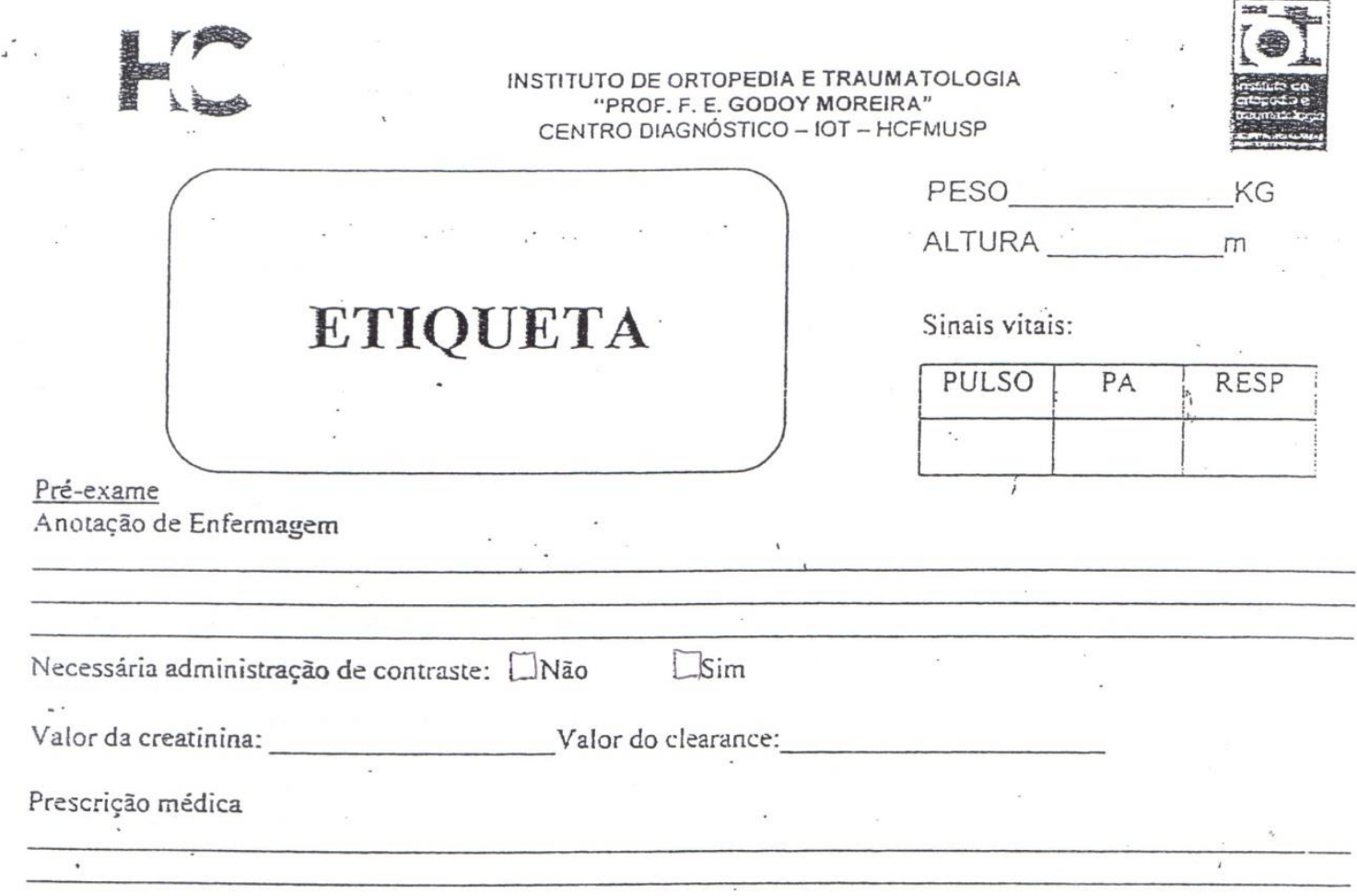

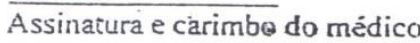

Check List Procedimento Seguro

\begin{tabular}{|c|c|c|c|}
\hline Confirmado paciente certo ( pulseira e prontuèrio) & $\square \sin$ & पNào & - \\
\hline Confirmado procedimento/Pedido certo & $\operatorname{Sim}$ & T] Não & \\
\hline Confirmado posicionamento certo & DS Sim & Nào & 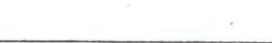 \\
\hline Confirmada Lateralidade & D & $\varepsilon$ & Q Nào se Aplica \\
\hline Confimado Jejum & DS Sim & ¿Não & Q Niàn se Aplica \\
\hline Confirmada Alergia: & $\square \operatorname{sim}$ & Não. & Qual? \\
\hline Confirmado identificação dù trascos de coleta * & ¿ $\sin$ & Não & {$[$ Niãos= Aplica } \\
\hline Confirmato materiais c eouipamentos ** & $\square_{\operatorname{sim}}$ & $\sum_{\text {Nào }}$ & D Nãoste Anlica \\
\hline Contimado paciente correto no Worklist (KISIPACS) & $\square \sin$ & DNào & \\
\hline
\end{tabular}

Astatura catimbo da Enfermatgem
Assinatura cuarimbo do
Técricol Biomédico
Assinaturate carimion do

Anestesisia 


\section{Questionário de segurança do paciente -RMI}

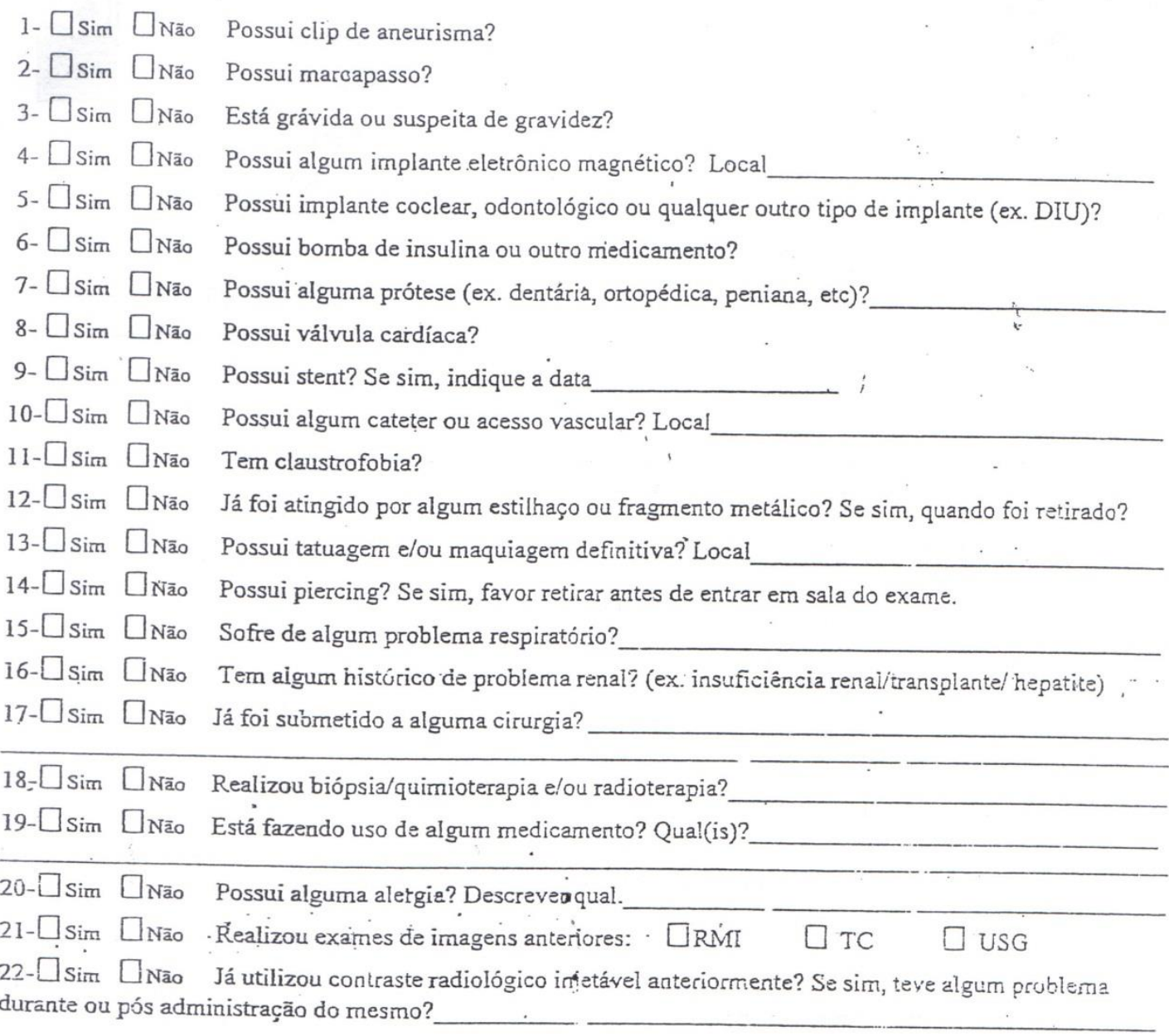

Declaro que fui informado(a) claramente sobre os riscos da utilização dos meios de contrastes radiológicos injetáveis e ( )autorizo/ () não autorizo de forma voluntária a realização do exame solicitado. $\therefore$

Assinatura do paciente'responsivel

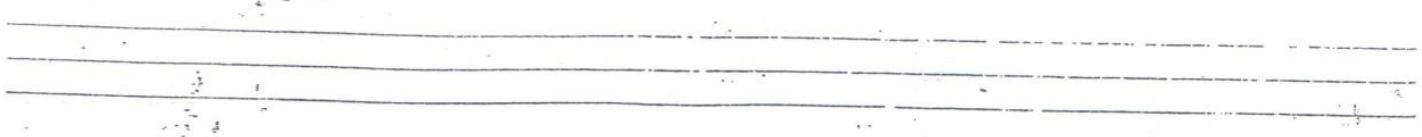




\section{ANEXO III - QUESTIONÁRIO - EXAME DE MEMBROS INFERIORES}

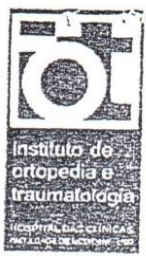

INSTIUTO OE ORTOPEDIA E TRAUMATOLOGIA SERVÇO DE DIAGNOSTCO POR IMAGEAR - IOT - HCFMUS

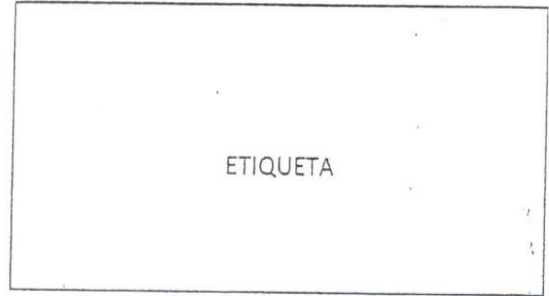

QUESTIONÁRIO - EXAME DE MEMBROS INFERIORES

\begin{tabular}{|c|c|c|c|}
\hline & $\square$ Coxa & $\square$ Perna & $\square$ Ambos \\
\hline 1 - Lateralidade: & $\square$ Direito & $\square$ Esquerdo & \\
\hline
\end{tabular}

3 - Já foi submetida a alguma cirurgia elou biópsia? Se sim, por favor, descreva abaixo.

4 - Já fez algum tratamento de quimioterapia ou radioterapia? Se sim, por favor, explique.

5 - Pratica algüm tipo de esporte?

6 - Teve algum trauma, infecçăo, torção ou fratura? Se sim, por favor, descreva abaixo.

7 - É diabético?

8 - Possui alguma lesão cutânea? (Marcar os locais no boneco abaixo).

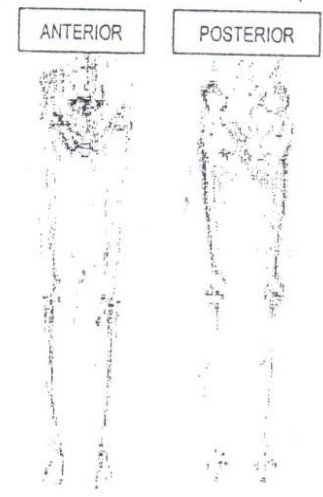

Questionário revisado por:

Biomédico (a) 
ANEXO IV - QUESTIONÁRIO INTERNACIONAL DE ATIVIDADE FÍSICA

- Forma Longa -

Nome: Data: ___ _ _ _

Idade: Sexo: F ( ) M ( ) Você trabalha de forma remunerada: ( ) Sim ( ) Não.

Quantas horas você trabalha por dia:

Quantos anos completos você estudou:

De forma geral sua saúde está:( ) Excelente ( ) Muito boa ( ) Boa ( ) Regular ( )Ruim

Nós estamos interessados em saber que tipos de atividade física as pessoas fazem como parte do seu dia a dia. Este projeto faz parte de um grande estudo que esta sendo feito em diferentes partes ao redor do mundo. Suas respostas nos ajudarão a entender que tão ativos nás somos em relção às pessoas de outros países. As perguntas estão relacionadas ao tempo que você gasta fazendo atividade física em uma semana NORMAL USUAL ou HABITUAL. As perguntas incluem as atividades que você faz no trabalho, para ir de um lugar a outro, por lazer, por esporte, por exercício ou como parte das suas atividades em casa ou no jardim. Suas respostas são MUlTO importantes. Por favor responda cada questão mesmo que considere que não seja ativo. Obrigado pela sua participação!

Para responder as questões lembre que:

- atividades físicas VIGOROSAS sao aquelas que precisam de um grande esforço físico e que fazem respirar MUITO mais forte que o normal

- atividades físicas MODERADAS sao aquelas que precisam de algum esforço físico e que fazem respirar UM POUCO mais forte que o normal

Esta seção inclui as atividades que você faz no seu serviço, que incluem trabalho remunerado ou voluntário, as atividades na escola ou faculdade e outro tipo de trabalho não remunerado fora da sua casa. NÃO incluir trabalho não remunerado que você faz na sua casa como tarefas domésticas, cuidar do jardim e da casa ou tomar conta da sua família. Estas serão incluídas na Seção 3. 
1a. Atualmente você trabalha ou faz trabalho voluntário fora de sua casa?

( ) Sim ( ) Não - Caso você responda não Vá para seção 2: Transporte

As próximas questões são em relação a toda a atividade física que você faz em uma semana USUAL ou NORMAL como parte do seu trabalho remunerado ou não remunerado. NÃO inclua o transporte para o trabaIho. Pense unicamente nas atividades que você faz por pelo menos 10 minutos contínuos:

1b. Em quantos dias de uma semana normal você gasta fazendo atividades vigorosas por pelo menos 10 minutos contínuos, como trabalho de construção pesada, carregar grandes pesos, trabalhar com enxada, escavar ou subir escadas como parte do seu trabalho: dias por SEMANA ( ) nenhum - Vá para a questão 1d.

1c. Quanto tempo no total você usualmente gasta POR DIA fazendo atividades físicas vigorosas como parte do seu trabalho?

horas minutos

1d. Em quantos dias de uma semana normal você faz atividades moderadas, por pelo menos 10 minutos contínuos, como carregar pesos leves como parte do seu trabalho ? dias por SEMANA ( ) nenhum - Vá para a questão 1f.

1e. Quanto tempo no total você usual mente gasta POR DIA fazendo atividades moderadas como parte do seu trabalho ? horas minutos

1f. Em quantos dias de uma semana normal você anda, durante pelo menos 10 minutos contínuos, como parte do seu trabalho? Por favor NÃO inclua o andar como forma de transporte para ir ou voltar do trabalho dias por SEMANA ( ) nenhum - Vá para a seção 2 - Transporte.

1g. Quanto tempo no total você usualmente gasta POR DIA caminhando como parte do seu trabalho? horas minutos 
Estas questões se referem a forma típica como você se desloca de um lugar para outro, incluindo seu trabalho, escola, cinema, lojas e outros.

2a. Em quantos dias de uma semana normal você anda de carro, ônibus, metrô ou trem? dias par SEMANA ( ) nenhum - Vá para questão 2c.

2b. Quanta tempo no total você usualmente gasta POR DIA andando de carro, ônibus, metrô ou trem?

horas minutos

2c. Em quantos dias de uma semana normal você anda de bicicleta por pelo menos 10 minutos contínuos para ir de um lugar para outro? (NÃO inclua o pedalar por lazer ou exercício) dias por SEMANA ( ) Nenhum - Vá para a questão 21.

2d. Nos dias que você pedal a quanta tempo no total você pedala POR DIA para ir de um lugar para outro? horas minutos

2e. Em quantos dias de uma semana normal você caminha por pelo menos 10 minutos contínuos para ir de um lugar para outro? (NÃO inclua as caminhadas por lazer ou exercício) dias por SEMANA ( ) Nenhum - Vá para a Seção 3.

21. Quando você caminha para ir de um lugar para outro quanta tempo POR DIA você gasta? (NÃO inclua as caminhadas por lazer ou exercício) horas minutos

SEÇÃO 3 - ATIVIDADE FÍSICA EM CASA: TRABALHO, TAREFAS DOMÉSTICAS E CUIDAR DA FAMÍLIA

Esta parte inclui as atividades físicas que você faz em uma semana NORMAL na sua casa e ao redor da sua casa, por exemplo trabalho em casa, cuidar do jardim, cuidar do quintal, trabalho 
de manutenção da casa ou para cuidar da sua família. Novamente pense somente naquelas atividades físicas que você faz por pelo menos 10 minutos contínuos.

3a. Em quantos dias de uma semana normal você faz atividades físicas vigorosas no jardim ou quintal por pelo menos 10 minutos como carpir, lavar o quintal, esfregar o chão: dias por SEMANA ( ) Nenhum - Vá para a questão 3c.

3b. Nos dias que você faz este tipo de atividades vigorosas no quintal ou jardim, quanto tempo no total você gasta POR DIA? horas minutos

3c . Em quantos dias de uma semana normal você faz atividades moderadas por pelo menos 10 minutos como carregar pesos leves, limpar vidros, varrer, rastelar com no jardim ou quintal dias por SEMANA ( ) Nenhum - Vá para questão 3e.

3d. Nos dias que você faz este tipo de atividades quanto tempo no total você gasta POR DIA fazendo essas atividades moderadas no jardim ou no quintal? horas minutos

3e. Em quantos dias de uma semana normal você faz atividades moderadas por pelo menos 10 minutos como carregar pesos leves, limpar vidros, varrer ou limpar o chão dentro da sua casa. dias por SEMANA ( ) Nenhum - Vá para seção 4.

3f. Nos dias que você faz este tipo de atividades moderadas dentro da sua casa quanto tempo no total você gasta POR DIA?

horas minutos

SEÇÃO 4 - ATIVIDADES FÍSICAS DE RECRAÇÃO, ESPORTE, DE EXERCÍCIO E DE LAZER

Esta seção se refere as atividades físicas que você faz em uma semana NORMAL unicamente por recreação, esporte, exercício ou lazer. Novamente pense somente nas atividades físicas que 
faz por pelo menos 10 minutos contínuos. Por favor NÃO inclua atividades que você ja tenha citado.

4a. Sem contar qualquer caminhada que você tenha citado anteriormente, em quantos dias de uma semana normal, você caminha por pelo menos 10 minutos contínuos no seu tempo livre? dias por SEMANA ( ) Nenhum - Vá para questão 4d.

4b. Nos dias em que você caminha no seu tempo livre, quanta tempo no total você gasta POR DIA?

horas minutos

4c. Em quantos dias de uma semana normal, você faz atividades vigorosas no seu tempo livre por pelo menos 10 minutos, como correr, fazer aeróbicos, nadar rápido, pedalar rápido ou fazer jogging:

dias por SEMANA ( ) Nenhum - Vá para questão 4f.

4e. Nos dias em que você faz estas atividades vigorosas no seu tempo livre quanto tempo no total você gasta POR DIA?

horas minutos

4f. Em quantos dias de uma semana normal, você faz atividades moderadas no seu tempo livre por pelo menos 10 minutos, como pedalar ou nadar a velocidade regular, jogar bola, volei, basquete, tênis : dias por SEMANA ( ) Nenhum - Vá para sessão 5.

4g. Nos dias em que você faz estas atividades moderadas no seu tempo livre quanta tempo no total você gasta POR DIA?

horas minutos

\section{SEÇÃO 5 - TEMPO GASTO SENTADO}

Estas últimas questões são sobre o tempo que você permanece sentado todo dia, no trabalho, na escola ou faculdade, em casa e durante seu tempo livre. Isto inciui o tempo sentado estudando, sentado enquanto descansa, fazendo lição de casa visitando um amigo, lendo, sentado ou 
deitado assistindo TV. NÃO inclua o tempo gasto sentando durante o transporte em ônibus, trem, metrô ou carro.

5a.Quanto tempo no total você gasta sentado durante um dia de semana? horas minutos

5b. Quanto tempo no total você gasta sentado durante em um dia de final de semana? horas minutos 